\title{
UNIQUENESS OF SOLUTIONS OF THE KDV-HIERARCHY VIA DUBROVIN-TYPE FLOWS
}

\author{
MILIVOJE LUKIĆ, GIORGIO YOUNG
}

\begin{abstract}
We consider the Cauchy problem for the KdV hierarchy - a family of integrable PDEs with a Lax pair representation involving one-dimensional Schrödinger operators - under a local in time boundedness assumption on the solution. For reflectionless initial data, we prove that the solution stays reflectionless. For almost periodic initial data with absolutely continuous spectrum, we prove that under Craig-type conditions on the spectrum, Dirichlet data evolve according to a Lipschitz Dubrovin-type flow, so the solution is uniquely recovered by a trace formula. This applies to algebro-geometric (finite gap) solutions; more notably, we prove that it applies to small quasiperiodic initial data with analytic sampling functions and Diophantine frequency. This also gives a uniqueness result for the Cauchy problem on the line for periodic initial data, even in the absence of Craig-type conditions.
\end{abstract}

\section{Introduction And Main Results}

The KdV equation

$$
\partial_{t} q+\frac{1}{4} \partial_{x}^{3} q-\frac{3}{2} q \partial_{x} q=0
$$

was studied in the 19th century by Boussinesq [4] and Korteweg-de Vries [24] as a model for solitary wave phenomena. Its modern theory began in the 1960s with works of Gardner-Greene-KruskalMiura [21] and Lax [26], who discovered that the KdV equation has infinitely many conserved quantities and can be formally rephrased in a Lax pair representation and studied through the associated family of one-dimensional Schrödinger operators. The conserved quantities formally correspond to mutually commuting Hamiltonians; this makes the KdV equation one of an infinite family of mutually commuting partial differential equations, called the KdV hierarchy.

The KdV hierarchy is most concisely introduced as the sequence of partial differential equations

$$
\frac{\partial q}{\partial t}=2 \frac{\partial \hat{f}_{n+1}}{\partial x}, \quad(x, t) \in \mathbb{R} \times \mathbb{R}
$$

where the $\hat{f}_{n}$ are differential polynomials in $q$, defined recursively by

$$
\begin{aligned}
& \hat{f}_{0}=1, \quad \hat{f}_{1}=q / 2, \\
& \hat{f}_{\ell+1}=-\frac{1}{2} \sum_{k=1}^{\ell} \hat{f}_{k} \hat{f}_{\ell+1-k}+\frac{1}{2} \sum_{k=0}^{\ell}\left(q \hat{f}_{k} \hat{f}_{\ell-k}+\frac{1}{4} \frac{\partial \hat{f}_{k}}{\partial x} \frac{\partial \hat{f}_{\ell-k}}{\partial x}-\frac{1}{2} \frac{\partial^{2} \hat{f}_{k}}{\partial x^{2}} \hat{f}_{\ell-k}\right) .
\end{aligned}
$$

Note that setting $n=0$ in (1.2) gives the translation flow $\partial_{t} q=\partial_{x} q$ and setting $n=1$ recovers the $\mathrm{KdV}$ equation (1.1). For $n \geq 1$, the $n$-th equation is a nonlinear partial differential equation with spatial derivatives up to order $2 n+1$, called the $\mathrm{KdV}-n$ equation. Due to the Lax pair representation, these PDEs are said to be integrable; 50 years after this discovery, many other integrable PDEs have been discovered, and $\mathrm{KdV}$ continues to be one of the central models on which new integrability phenomena are explored [16].

M.L. was supported in part by NSF grant DMS-1700179.

G.Y. was supported in part by NSF grant DMS-1745670. 
Of course, rigorous results about the $\mathrm{KdV}$ equation and hierarchy are dependent on the type of initial data being considered,

$$
q(x, 0)=V(x) .
$$

The first application of the Lax pair representation was to rapidly decaying initial data via the inverse scattering transform of Gelfand-Levitan-Marchenko. Other classical applications were to periodic and finite gap quasiperiodic initial data; in those applications, solutions can be parametrized by Dirichlet data, which evolve by Dubrovin-type flows, or by an Abel map which linearizes the trajectories on a torus. In particular, the finite gap quasiperiodic solutions are described by an algebro-geometric approach associated to a compact Riemann surface. The solutions are quasiperiodic in both space and time because they can be expressed in the form $q(x, t)=F(\delta x+\zeta t)$ where $F: \mathbb{T}^{d} \rightarrow \mathbb{R}$ is continuous and $\delta, \zeta \in \mathbb{R}^{d}$, where $d<\infty$ is the number of gaps.

These theories motivate a more general consideration of the KdV hierarchy with almost periodic initial data. Recall that a function $f: \mathbb{R} \rightarrow \mathbb{C}$ is called almost periodic if the set of its translates $\{f(\cdot-s) \mid s \in \mathbb{R}\}$ is precompact in the uniform norm; this includes continuous periodic and quasiperiodic functions as special cases. In the study of the KdV hierarchy with almost periodic initial data, one of the new difficulties is in the nature of the conserved quantities which take the form of spatial averages over $\mathbb{R}$ such as $\lim _{L \rightarrow \infty} \frac{1}{2 L} \int_{-L}^{L} q(x, t)^{2} d x$, which are not useful for obtaining local control over the solution. Indeed, even short time existence of solutions is not known for arbitrary almost periodic initial data. From a spectral/scattering perspective, the difficulty comes from the fact that almost periodic Schrödinger operators have very diverse spectral properties.

Despite these obstacles, the $\mathrm{KdV}$ hierarchy with almost periodic initial data is an active area of research [17, 18, 38, 9, 2, 19, motivated by a question of Deift [14, 15] about whether such solutions are almost periodic in time. Most of these works are based on the reflectionless property and on spectral theoretic techniques initially developed in the time-independent setting (i.e. for the translation flow) [6, 33, 34]. Analogous questions have also been studied in the setting of the nonlinear Schrödinger equation and the Toda lattice [28, 5, 39, 3].

To get more precise, we must recall some basic facts about Schrödinger operators, starting with a time-independent setting. We denote by $\mathrm{AC}_{\text {loc }}(\mathbb{R})$ the set of functions on $\mathbb{R}$ which are absolutely continuous on every compact interval in $\mathbb{R}$; such functions have a derivative in $L_{\text {loc }}^{1}(\mathbb{R})$. We can describe in those terms the familiar Sobolev space

$$
W^{2,2}(\mathbb{R})=\left\{f \in L^{2}(\mathbb{R}) \mid f \in \mathrm{AC}_{\mathrm{loc}}(\mathbb{R}), f^{\prime} \in \mathrm{AC}_{\mathrm{loc}}(\mathbb{R}), f^{\prime \prime} \in L^{2}(\mathbb{R})\right\}
$$

For a bounded function $W: \mathbb{R} \rightarrow \mathbb{R}$, we consider the Schrödinger operator

$$
H_{W}=-\frac{\mathrm{d}^{2}}{\mathrm{~d} x^{2}}+W(x)
$$

which is an unbounded self-adjoint operator on $L^{2}(\mathbb{R})$ with the domain $W^{2,2}(\mathbb{R})$. In particular, $\sigma\left(H_{W}\right) \subset \mathbb{R}$. For any $z \in \mathbb{C} \backslash \sigma\left(H_{W}\right)$, the resolvent operator $\left(H_{W}-z\right)^{-1}$ exists and is an integral operator; its integral kernel is called the Green's function and denoted $G(x, y ; z, W)$. Of particular interest is the diagonal Green's function, obtained by setting $y=x$. This is a Herglotz function (an analytic function which maps $\mathbb{C}_{+}$to itself) so it has nontangential boundary values Lebesgue-a.e. on $\mathbb{R}$. The Schrödinger operator $H_{W}$ is called reflectionless if

$$
\operatorname{Re} G(x, x ; \lambda+i 0, W)=0 \text {, for Lebesgue a.e. } \lambda \in \sigma\left(H_{W}\right) .
$$

Let us denote by $\mathcal{R}(S)$ the set of bounded potentials $W$ such that $H_{W}$ is reflectionless and $\sigma\left(H_{W}\right)=$ $S$; we make $\mathcal{R}(S)$ a metric space with the $L^{\infty}$-metric.

Our first result is that under a boundedness assumption on solutions to $\mathrm{KdV}-n$, the reflectionless property is preserved in time; this generalizes or extends previous results for the KdV equation in [30, 29, 2]. 
Proposition 1.1. Let $q(x, t)$ be a classical solution to the Cauchy problem (1.2), (1.3) on $x \in \mathbb{R}$, $t \in[0, T]$ for some $T<\infty$, obeying the boundedness condition

$$
q, \partial_{x}^{2 n} q \in L^{\infty}(\mathbb{R} \times[0, T]) .
$$

Let $S=\sigma\left(H_{V}\right)$. If $V \in \mathcal{R}(S)$, then $q(\cdot, t) \in \mathcal{R}(S)$ for all $t \in[0, T]$.

In the above, we consider classical solutions $q$ of (1.2), i.e., solutions such that $q$ is $2 n+1$ times differentiable in $x, \partial_{x}^{2 n+1} q$ is jointly continuous in $x$ and $t, q$ is differentiable in $t$ and (1.2) holds pointwise.

Periodic potentials are usually treated through a well-posedness theory in Sobolev spaces $H^{\beta}(\mathbb{T})$, and described in terms of a nonlinear evolution map $\mathcal{S}_{t}: H^{\beta}(\mathbb{T}) \rightarrow H^{\beta}(\mathbb{T})$ which is a homeomorphism for each $t$ and generates solutions $q(\cdot, t)=\mathcal{S}_{t}(V)$ of $\mathrm{KdV}-n$. Built into that formulation is the a priori assumption that solutions $q(x, t)$ stay periodic in $x$ for all $t$. In contrast, Prop. 1.1 allows us to consider locally bounded solutions and actually recover spatial periodicity for periodic intial data. Indeed, we have the following corollary as a consequence of Prop. 1.1.

Corollary 1.2. Let $q(x, t)$ be a classical solution to the Cauchy problem (1.2), (1.3) on $x \in \mathbb{R}$, $t \in[0, T]$ for some $T<\infty$, obeying the boundedness condition (1.6). Suppose that the initial data satisfies $V(x+\ell)=V(x)$ for an $\ell>0$ and all $x \in \mathbb{R}$. Then, for all $t \in[0, T], x \in \mathbb{R}$, $q(x+\ell, t)=q(x, t)$ and $q(\cdot, t)=\mathcal{S}_{t}(V)$.

Note that since our result is about uniqueness, finiteness of $T$ in the previous theorem should be viewed as a strength, not a weakness; it immediately implies the following result for global solutions.

Corollary 1.3. Let $q(x, t)$ be a classical solution to the Cauchy problem (1.2), (1.3) on $x \in \mathbb{R}$, $t \in[0, \infty)$, obeying the boundedness condition (1.6) for all $T<\infty$. Suppose that the initial data satisfies $V(x+\ell)=V(x)$ for an $\ell>0$ and all $x \in \mathbb{R}$. Then, for all $t \in[0, \infty), x \in \mathbb{R}, q(x+\ell, t)=$ $q(x, t)$ and $q(\cdot, t)=\mathcal{S}_{t}(V)$.

After these results for the periodic problem, we will return to the more general setting, inspired by the regime of almost periodic initial data. We recall an important link between the reflectionless property and almost periodicity: by Kotani theory [25], if $W$ is almost periodic and its absolutely continuous spectrum is $\sigma_{\mathrm{ac}}\left(H_{W}\right)=\sigma\left(H_{W}\right)$, then $H_{W}$ is reflectionless. We will use this in an application of our results in the final section of the paper.

In the nonperiodic setting, our work will use certain thickness conditions on the spectrum. By general principles, $S=\sigma\left(H_{W}\right)$ is a closed set bounded below but not bounded above, so it can be written in the form

$$
S=[\underline{E}, \infty) \backslash \cup_{j \in J}\left(E_{j}^{-}, E_{j}^{+}\right),
$$

where $\underline{E}=\inf S, J$ is a countable indexing set and $\left(E_{j}^{-}, E_{j}^{+}\right)$denote maximal open intervals in $[\underline{E}, \infty) \backslash S$ called gaps. The "finite gap" solutions correspond to sets $S$ with finitely many gaps, and periodic potentials have a sequence of isolated gaps indexed by $n \in \mathbb{N}$ such that $E_{n}^{ \pm} \rightarrow+\infty$ as $n \rightarrow \infty$; however, for general almost periodic initial data the set $S$ is generically a Cantor set and can even have zero Lebesgue measure [1, 7]. Many difficulties arise from the possible accumulation of gaps at finite points.

Using the observation that $G(x, x ; z, W)$ is continuous and strictly increasing for $z \in\left(E_{j}^{-}, E_{j}^{+}\right)$, Craig [6] introduced Dirichlet data for $W \in \mathcal{R}(S)$ by

$$
\mu_{j}(x)= \begin{cases}z \in\left(E_{j}^{-}, E_{j}^{+}\right), & G(x, x ; z, W)=0, \\ E_{j}^{+}, & G(x, x ; z, W)<0 \forall z \in\left(E_{j}^{-}, E_{j}^{+}\right), \\ E_{j}^{-}, & G(x, x ; z, W)>0 \forall z \in\left(E_{j}^{-}, E_{j}^{+}\right) .\end{cases}
$$


If $\mu_{j}(x) \in\left(E_{j}^{-}, E_{j}^{+}\right)$, additional information is in the value of $\sigma_{j}(x)=-\left.\partial_{x} G(x, x ; z, W)\right|_{z=\mu_{j}(x)} \in$ $\{ \pm 1\}$. Together, for fixed $x \in \mathbb{R}, \mu_{j}(x)$ and $\sigma_{j}(x)$ can be thought of as lying on a double cover of the interval $\left[E_{j}^{-}, E_{j}^{+}\right]$with gap edges identified, and they can be combined into an angular variable $\varphi_{j}(x) \in \mathbb{R} / 2 \pi \mathbb{Z}$ defined by the conditions

$$
\begin{aligned}
& \mu_{j}(x)=E_{j}^{-}+\left(E_{j}^{+}-E_{j}^{-}\right) \cos ^{2}\left(\frac{\varphi_{j}(x)}{2}\right), \\
& \sigma_{j}(x)=-\operatorname{sgn} \sin \varphi_{j}(x) .
\end{aligned}
$$

We view this as a correspondence from $W \in \mathcal{R}(S)$ to a trajectory $\varphi(x)=\left(\varphi_{j}(x)\right)_{j \in J}$ on the "torus of Dirichlet data" $\mathcal{D}(S):=\mathbb{T}^{J}$.

In particular, the map $W \mapsto \varphi(0)$ is a map from $\mathcal{R}(S)$ to $\mathcal{D}(S)$, which will be a homeomorphism in our regime. The recovery of $W$ from its Dirichlet data $\varphi(0)$ uses the behavior of Dirichlet data with respect to translation: it was proven in [6, 2] that the translation flow is governed by the ordinary differential equation

$$
\partial_{x} \varphi(x)=\Psi(\varphi(x)),
$$

where $\Psi$ is the Dubrovin-type vector field on $\mathcal{D}(S)$ with components

$$
\Psi_{j}(\varphi)=2 \sqrt{\left(\mu_{j}-\underline{E}\right) \prod_{\ell \neq j} \frac{\left(E_{\ell}^{-}-\mu_{j}\right)\left(E_{\ell}^{+}-\mu_{j}\right)}{\left(\mu_{\ell}-\mu_{j}\right)^{2}}} .
$$

Under suitable thickness assumptions on the spectrum, which will be discussed below, Craig [6] proved that $\Psi$ is Lipschitz, so the solution to the ODE (1.11) is unique for any given initial data $\varphi(0) \in \mathcal{D}(S)$. This trajectory $\varphi(x)$ determines the potential by (1.9) and the trace formula

$$
W(x)=\underline{E}+\sum_{j \in J}\left(E_{j}^{-}+E_{j}^{+}-2 \mu_{j}(x)\right)
$$

which holds in great generality [23]. In summary, the map from $\mathcal{R}(S)$ to $\mathcal{D}(S)$ given by $W \mapsto \varphi(0)$ is then a homeomorphism, with an explicit inverse given by solving the ODE (1.11) and applying the trace formula (1.13).

For the set $S$ with gaps denoted as in (1.7), we denote

$$
\begin{aligned}
\gamma_{j} & =E_{j}^{+}-E_{j}^{-}, \\
\eta_{j, l} & =\operatorname{dist}\left(\left(E_{j}^{-}, E_{j}^{+}\right),\left(E_{l}^{-}, E_{l}^{+}\right)\right), \\
\eta_{j, 0} & =\operatorname{dist}\left(\underline{E},\left(E_{j}^{-}, E_{j}^{+}\right)\right), \\
C_{j} & =\left(\eta_{j, 0}+\gamma_{j}\right)^{1 / 2} \prod_{\substack{l \in J \\
l \neq j}}\left(1+\frac{\gamma_{l}}{\eta_{j, l}}\right)^{1 / 2} .
\end{aligned}
$$

To study the KdV- $n$ equation, we fix $n$ and assume that $S$ obeys the moment condition

$$
\sum_{k \in J} \gamma_{k}\left(1+\eta_{k, 0}^{n}\right)<\infty
$$

and we set the metric on $\mathcal{D}(S)$ given by

$$
\|\varphi-\tilde{\varphi}\|_{\mathcal{D}(S)}=\sup _{j \in J} \gamma_{j}^{1 / 2}\left(1+\eta_{j, 0}^{n}\right)^{1 / 2}\left\|\varphi_{j}-\tilde{\varphi}_{j}\right\|_{\mathbb{T}}
$$

which is consistent with the product topology. In particular, the torus $\mathcal{D}(S)$ is compact. 
We consider the scalar fields on $\mathcal{D}(S)$

$$
Q_{k}=\underline{E}^{k}+\sum_{j \in J}\left(\left(E_{j}^{-}\right)^{k}+\left(E_{j}^{+}\right)^{k}-2 \mu_{j}^{k}\right)
$$

and

$$
R_{m}=\sum_{\substack{\alpha \in \mathbb{N}_{0}^{n} \\ \sum_{k=1}^{m} k \alpha_{k}=m}} \prod_{k=1}^{m} \frac{Q_{k}^{\alpha_{k}}}{\alpha_{k} !(2 k)^{\alpha_{k}}} .
$$

It will follow from (1.15) that $Q_{k}$ and thus $R_{m}$ are continuous scalar fields for all $k, m \leq n$.

We also consider another Dubrovin-type vector field $\Xi$ with components

$$
\Xi_{j}(\varphi)=\left(\sum_{\ell=0}^{n} R_{n-\ell} \mu_{j}^{\ell}\right) \Psi_{j} .
$$

We formulate conditions to ensure that the vector field $\Xi$ is Lipschitz on $\mathcal{D}(S)$. These Craig-type conditions are as follows,

$$
\begin{gathered}
\sum_{k \in J} \gamma_{k}^{1 / 2}\left(1+\eta_{k, 0}^{n}\right)^{1 / 2}<\infty, \\
\sup _{j \in J} C_{j}\left(1+\eta_{j, 0}^{n}\right)^{3 / 2} \sum_{k \neq j} \frac{\gamma_{k}^{1 / 2} \gamma_{j}^{1 / 2}}{\eta_{j, k}}<\infty, \\
\sup _{j \in J} \frac{\gamma_{j}\left(1+\eta_{j, 0}^{n}\right) C_{j}}{\eta_{j, 0}}<\infty, \\
\sup _{j \in J} C_{j} \gamma_{j}^{1 / 2}\left(1+\eta_{j, 0}^{n}\right)^{3 / 2}<\infty,
\end{gathered}
$$

with $C_{j}$ given by (1.14). Although spectral gaps can accumulate at a finite point, these conditions provide quantitative bounds on the accumulation of gaps and serve as thickness conditions on the spectrum. (1.20) is a kind of moment condition and it implies (1.15).

As in the discussion surrounding (1.11), by [6, 2], the translation flow is governed by the following ordinary differential equation for fixed $t$,

$$
\partial_{x} \varphi(x, t)=\Psi(\varphi(x, t)) .
$$

It was also proven in [6] that $\Psi$ is Lipschitz under weaker assumptions than (1.20), (1.21), (1.22), and (1.23). In this paper we will show that

$$
\partial_{t} \varphi(x, t)=\Xi(\varphi(x, t))
$$

and that $\Xi$ is Lipschitz under assumptions (1.20), (1.21), (1.22), and (1.23). Note that the Craigtype conditions (1.20), (1.21), (1.22), (1.23) are inevitably stronger than those in 6] (which were designed to control the translation flow $\Psi$ ) or those in [2] (designed to control the flow corresponding to the $\mathrm{KdV}$ equation, $n=1$ ). The Lipschitz property will imply that the two-parameter solution $\varphi(x, t)$ solving (1.24) and (1.25) is uniquely determined by $\varphi(0,0)$, which is in turn uniquely determined by the initial data $V$. We prove the following:

Theorem 1.4. Let $q(x, t)$ be a classical solution to (1.2), (1.3) on $x \in \mathbb{R}, t \in[0, T]$ for some $T<\infty$, with initial data $V$ such that $H_{V}$ is reflectionless and the spectrum $S=\sigma\left(H_{V}\right)$ obeys (1.20), (1.21), (1.22), (1.23). We also assume $q$ obeys (1.6). Then, for all $t \in[0, T]$, 
(a) The space and time evolution of Dirichlet data $\varphi=\left(\varphi_{j}\right)_{j \in J}$ corresponding to $q(x, t)$ is uniquely determined from $\varphi(0,0)$ as the solution of the flows (1.24), (1.25) with respect to the Lipshitz vector fields $\Psi$ and $\Xi$.

(b) The solution $q(x, t)$ obeys

$$
q(x, t)=\underline{E}+\sum_{j \in J}\left(E_{j}^{+}+E_{j}^{-}-2 \mu_{j}(x, t)\right)
$$

where $\sigma\left(H_{V}\right)=[\underline{E}, \infty) \backslash \bigcup_{j \in J}\left(E_{j}^{-}, E_{j}^{+}\right)$and $\mu_{j}(x, t)$ is the Dirichlet data.

(c) In particular, $q$ is uniquely determined by (1.25) and (1.26).

Again, we have the following result for global solutions.

Corollary 1.5. Let $q(x, t)$ be a classical solution to (1.2), (1.3) on $x \in \mathbb{R}, t \in[0, \infty)$, with initial data $V$ such that $H_{V}$ is reflectionless and the spectrum $S=\sigma\left(H_{V}\right)$ obeys (1.20), (1.21), (1.22), and (1.23). If $q$ obeys the boundedness condition (1.6) for all $T<\infty$, then the conclusions of the previous theorem apply to $q$ for all $t \in[0, \infty)$.

Analogous results hold for negative time.

It should be noted that Theorem 1.4 doesn't assume that our solution stays almost periodic in $x$; instead, this follows as a consequence. In this way, in addition to saying something new for the periodic case as we highlighted in Corollary 1.2, we also see that our results apply to the finite gap quasiperiodic case (where the Craig-type conditions are trivially satisfied). The theorem also applies to a class of small quasiperiodic initial data which we will describe below.

An earlier uniqueness result for the KdV equation was proved by Binder-Damanik-GoldsteinLukic [2] and is based on work of Rybkin [30] on the time evolution of Weyl solutions, $m$-functions and $M$-matrices under the KdV equation. Our paper can be viewed as a generalization of these results to the entire KdV hierarchy. Moreover, through a more careful analysis of the time evolution of eigensolutions, our results improve those in [30, 2] even for the KdV equation. Where earlier results for the $\mathrm{KdV}$ equation require $q, \partial_{x}^{3} q \in L^{\infty}(\mathbb{R} \times[0, T])$, our Theorem 1.4 for $n=1$ only requires $q, \partial_{x}^{2} q \in L^{\infty}(\mathbb{R} \times[0, T])$.

The paper [2] also proved existence and almost periodicity of solutions to the KdV equation for a class of reflectionless initial data with Craig-type conditions on the spectrum $S$; these results were generalized by Eichinger-VandenBoom-Yuditskii [19] to a more general class of $S$ (Widom sets with the Direct Cauchy Theorem property and a moment condition) and to the entire KdV hierarchy. Our uniqueness results can be viewed as complementary to those existence and almost periodicity results. Within the scope of applicability, our results show that the almost periodic solution constructed in [19] is the only locally bounded solution in the sense of (1.6).

Finally, we describe the application to small quasiperiodic initial data. Let $\epsilon>0,0 \leq \kappa_{0} \leq 1$, and $\omega \in \mathbb{R}^{\nu}$ for some $\nu \in \mathbb{N}$. We say $V \in \mathcal{P}\left(\omega, \epsilon, \kappa_{0}\right)$ if $V: \mathbb{R} \rightarrow \mathbb{R}$ is of the form

$$
V(x)=\sum_{m \in \mathbb{N}^{\nu}} c(m) e^{2 \pi i m \cdot \omega x}
$$

where

$$
|c(m)| \leq \epsilon \exp \left(-\kappa_{0}|m|\right), \forall n \in \mathbb{Z}^{\nu} .
$$

All results will be in the small coupling regime, $\epsilon<\epsilon_{0}\left(a_{0}, b_{0}, \kappa_{0}\right)$. The direct spectral theory of $H_{V}$ for $\epsilon$ has been studied extensively by Eliasson [20] and Damanik-Goldstein [8], with studies of the inverse spectral theory and the KdV equation in [38, 10, 11, 12, 2]. Using that theory, we will be able to prove that Theorem 1.4 applies to initial data $V \in \mathcal{P}\left(\omega, \epsilon, \kappa_{0}\right)$.

We now state our uniqueness theorem as applied to data of the form (1.27) above, with $\omega$ satisfying a Diophantine condition,

$$
|m \cdot \omega| \geq a_{0}|m|^{-b_{0}}
$$


for some $0<a_{0}<1$ and $\nu<b_{0}<\infty$.

Theorem 1.6. Let $\omega \in \mathbb{R}^{\nu}$ obey the Diophantine condition (1.28) for some $0<a_{0}<1$ and $\nu<b_{0}<\infty$. There is an $\epsilon_{0}\left(a_{0}, b_{0}, \kappa_{0}\right)>0$ such that if $\epsilon<\epsilon_{0}$, and $V \in \mathcal{P}\left(\omega, \epsilon, \kappa_{0}\right)$, then any solution of (1.2), (1.3), with $q, \partial_{x}^{2 n} q \in L^{\infty}(\mathbb{R} \times[0, T])$ is unique.

Acknowledgments. We would like to thank Benjamin Eichinger and Jo Nelson for clarifying conversations.

\section{Time evolution of the Weyl solutions and the Weyl $M$-matrix}

In this section, we will study the time dependence of Weyl solutions and the Weyl $M$-matrix for the family of Schrödinger operators associated to a fixed classical solution $q$ of (1.2), (1.3) which obeys the boundedness condition (1.6).

Historically, the discovery of the Lax pair representation was preceded by a description in [21] of the time-evolution of formal eigensolutions for the Schrödinger operator. The main analytical result of this section is that under suitable conditions, not only are eigensolutions preserved in this way, but so are Weyl solutions, as defined below. After describing the time evolution of Weyl solutions, we will be able to compute the time evolution of Weyl $m$-functions, the Weyl $M$-matrix, and reflection coefficients.

If $W: \mathbb{R} \rightarrow \mathbb{R}$ is bounded, the Schrödinger operator $H_{W}$ is in the "limit point" case at $\pm \infty$, i.e., for any $z \in \mathbb{C} \backslash \sigma\left(H_{W}\right)$ and each halfline $[0, \pm \infty)$, there is a one-dimensional subspace of solutions of

$$
-\psi^{\prime \prime}+W \psi=z \psi
$$

which are square-integrable on that half-line. Any nontrivial eigensolution which is square-integrable on the half-line $[0, \pm \infty)$ is called a Weyl solution at $\pm \infty$ and denoted by $\psi_{ \pm}(x ; W)$; Weyl solutions are defined up to normalization. It is a common convention to set $\psi_{ \pm}(0 ; W)=1$, but this convention isn't natural in time-dependent considerations or when changing reference points. Instead, we will assume that a Weyl solution has been chosen corresponding to $q(\cdot, 0)=V$ and will consider a time evolution for the eigensolution, in which both $x$-dependence and $t$-dependence are written as a first-order (system of) ODEs.

We define, as in [22],

$$
\begin{aligned}
Q(x, t) & =\left(\begin{array}{cr}
0 & 1 \\
q(x, t)-z & 0
\end{array}\right), \\
P(x, t) & =\left(\begin{array}{cc}
-\frac{1}{2} \partial_{x} \hat{F}_{n}(z) & \hat{F}_{n}(z) \\
(q-z) \hat{F}_{n}(z)-\frac{1}{2} \partial_{x}^{2} \hat{F}_{n}(z) & \frac{1}{2} \partial_{x} \hat{F}_{n}(z)
\end{array}\right),
\end{aligned}
$$

where

$$
\hat{F}_{n}(z)=\sum_{\ell=0}^{n} \hat{f}_{n-\ell} z^{\ell} .
$$

We consider, for any $z \in \mathbb{C} \backslash \sigma\left(H_{V}\right)$, the system of PDEs

$$
\left\{\begin{array}{l}
\partial_{t} \nu_{ \pm}(x, t)=P(x, t) \nu_{ \pm}(x, t) \\
\partial_{x} \nu_{ \pm}(x, t)=Q(x, t) \nu_{ \pm}(x, t) \\
\nu_{ \pm}(0,0)=\left(\begin{array}{l}
\psi_{ \pm}(0 ; V) \\
\psi_{ \pm}^{\prime}(0 ; V)
\end{array}\right)
\end{array}\right.
$$

$Q(x, t)$ is the standard matrix for converting the second order eigenvalue equation, $H_{q(\cdot, t)} \psi=z \psi$, to a first order system, and $P(x, t)$ will determine the time evolution for the Weyl solutions of $H_{V}$. 
The matrix functions $P, Q: \mathbb{R}^{2} \rightarrow M_{2}(\mathbb{C})$ satisfy the zero curvature condition if

$$
\partial_{t} Q-\partial_{x} P+[P, Q]=0
$$

where $[P, Q]$ is the commutator. As an equality of differential expressions, the zero curvature condition (2.3) is equivalent to (1.2) [22], and (2.3) is sometimes viewed as a different way to introduce the KdV hierarchy.

Remark 2.1. Existence of a joint solution of the system (2.2) depends on the zero curvature condition (2.3) by standard arguments from differential geometry. While these arguments are often presented under $C^{\infty}$ assumptions, they hold under our smoothness conditions. Namely, the zero curvature condition (2.3) involves $\partial_{x} P$, a differential polynomial with at most $2 n+1$ spatial derivatives of $q$ in each entry, and $\partial_{t} Q$, which includes only one time derivative of $q$ in the bottom left coordinate. For a classical solution $q$ of $\mathrm{KdV}-n$, these partial derivatives exist and are jointly continuous in $(x, t)$. The system (2.2) can be written as an autonomous system on $(x, t, \nu) \in \mathbb{R}^{2} \times \mathbb{C}^{2}$ corresponding to vector fields $\hat{Q}, \hat{P}$ written in the language of differential geometry as

$$
\begin{aligned}
& \hat{Q}:=\partial_{x}+P^{i j}(x, t) v_{j} \partial \nu_{i} \\
& \hat{P}:=\partial_{t}+Q^{i j}(x, t) v_{j} \partial \nu_{i} .
\end{aligned}
$$

These vector fields are $C^{1}$, since, as in the above, $P$ involves a differential polynomial with only $2 n$ spatial derivatives on $q$, while $Q$ only has $(x, t)$ dependence through $q$. Then (2.3) is equivalent to the vanishing of the Lie bracket $L_{\hat{P}} \hat{Q}=[\hat{P}, \hat{Q}]=0$, so the flows of $\hat{P}$ and $\hat{Q}$ commute by differential geometry arguments in [35, Lemma 5.13]. This shows existence of a solution of (2.2). Uniqueness follows from the fact that $\hat{Q}, \hat{P} \in C^{1}$.

We will now prove that the first component of the solution $\nu_{ \pm}(x, t)$ of (2.2) is the Weyl solution for $H_{q(\cdot, t)}$ for each $t \in[0, T]$.

Proposition 2.1. Let $q$ be a classical solution of the Cauchy problem (1.2), (1.3) obeying the boundedness condition (1.6). Fix $z \in \mathbb{C} \backslash \sigma\left(H_{V}\right)$ and let

$$
\nu_{ \pm}(x, t)=\left(\begin{array}{l}
\alpha_{ \pm}(x, t) \\
\beta_{ \pm}(x, t)
\end{array}\right)
$$

be the solution to (2.2). Then, for every $t \in[0, T], \alpha_{ \pm}(\cdot, t)$ is a Weyl solution at $\pm \infty$ for the potential $q(\cdot, t)$.

Proof. It follows from the form of $Q$ that $\partial_{x} \alpha_{ \pm}(x, t)=\beta_{ \pm}(x, t)$ and

$$
\partial_{x}^{2} \alpha_{ \pm}(x, t)=\partial_{x} \beta_{ \pm}(x, t)=(q(x, t)-z) \alpha_{ \pm}(x, t)
$$

so for each $t, \alpha_{ \pm}(x, t)$ as a function of $x$ solves the eigensolution equation for potential $q(\cdot, t)$. It remains to prove that it is nontrivial for each $t$ and square-integrable on the corresponding half-line.

Let us denote

$$
g(x, t):=\left|\alpha_{ \pm}(x, t)\right|^{2}+\left|\beta_{ \pm}(x, t)\right|^{2}=\left\|\nu_{ \pm}(x, t)\right\|_{\mathbb{C}^{2}}^{2} .
$$

Then

$$
\partial_{t} g(x, t)=2 \operatorname{Re}\left\langle\partial_{t} \nu_{ \pm}(x, t), \nu_{ \pm}(x, t)\right\rangle_{\mathbb{C}^{2}}=2 \operatorname{Re}\left\langle P(x, t) \nu_{ \pm}(x, t), \nu_{ \pm}(x, t)\right\rangle_{\mathbb{C}^{2}}
$$

so by the Cauchy-Schwarz inequality and using the operator norm of $P(x, t)$,

$$
\left|\partial_{t} g(x, t)\right| \leq 2\|P(x, t)\| g(x, t) .
$$

Since entries of $P(x, t)$ are polynomial expressions in $q, \partial_{x} q, \ldots, \partial_{x}^{2 n} q$, it follows from Sobolev inequalities and the boundedness assumption $q, \partial_{x}^{2 n} q \in L^{\infty}(\mathbb{R} \times[0, T])$ that

$$
C=\sup _{x \in \mathbb{R}} \sup _{t \in[0, T]}\|P(x, t)\|<\infty .
$$


Thus, it follows that

$$
g(x, 0) e^{-2 C t} \leq g(x, t) \leq g(x, 0) e^{2 C t}
$$

for $t \in[0, T]$ and therefore

$$
e^{-2 C t} \int_{0}^{ \pm \infty}\left\|\nu_{ \pm}(x, 0)\right\|^{2} d x \leq \int_{0}^{ \pm \infty}\left\|\nu_{ \pm}(x, t)\right\|^{2} d x \leq e^{2 C t} \int_{0}^{ \pm \infty}\left\|\nu_{ \pm}(x, 0)\right\|^{2} d x .
$$

Since $\alpha_{ \pm}(\cdot, 0)$ is a Weyl solution corresponding to the bounded potential $V$, it is by definition nontrivial. Moreover, by boundedness of $V$, the derivative of the Weyl solution is also squareintegrable on the corresponding half-line [36, 37, 31, 27], so

$$
0<\int_{0}^{ \pm \infty}\left\|\nu_{ \pm}(x, 0)\right\|^{2}<\infty .
$$

It follows from (2.4) that for all $t \in[0, T]$,

$$
0<\int_{0}^{ \pm \infty}\left\|\nu_{ \pm}(x, t)\right\|^{2} d x<\infty .
$$

Strict positivity implies that $\alpha_{ \pm}(\cdot, t)$ is nontrivial and finiteness that $\alpha_{ \pm}(\cdot, t)$ is the Weyl solution.

By the above lemma, we consider $\psi_{ \pm}(x, t ; z)=\alpha_{ \pm}(x, t ; z)$ a Weyl solution of $H(q(\cdot, t))$ at energy $z$ and we know that its time evolution is governed by the $P$-matrix,

$$
\partial_{t}\left(\begin{array}{c}
\psi_{ \pm}(x, t ; z) \\
\partial_{x} \psi_{ \pm}(x, t ; z)
\end{array}\right)=P(x, t ; z)\left(\begin{array}{c}
\psi_{ \pm}(x, t ; z) \\
\partial_{x} \psi_{ \pm}(x, t ; z)
\end{array}\right)
$$

Note that we are now making the $z$ dependence explicit in our notation.

In particular the Weyl $m$ functions are now defined uniquely (regardless of the choice of normalization of $\left.\psi_{ \pm}(x, 0, z)\right)$ as the logarithmic derivatives of the Weyl solutions,

$$
m_{ \pm}(x, t ; z)= \pm \frac{\partial_{x} \psi_{ \pm}(x, t ; z)}{\psi_{ \pm}(x, t ; z)}
$$

and (2.5) allows us to determine their time evolution, as well as that of the Weyl $M$-matrix,

$$
M=\left(\begin{array}{cc}
\frac{-1}{m_{-}+m_{+}} & \frac{1}{2} \frac{m_{-}-m_{+}}{m_{-}+m_{+}} \\
\frac{1}{2} \frac{m_{-}-m_{+}}{m_{-}+m_{+}} & \frac{m_{-} m_{+}}{m_{-}+m_{+}}
\end{array}\right)
$$

The following lemma follows an argument described in [30] for the KdV equation; we repeat the argument for the rest of the hierarchy for the sake of completeness.

Lemma 2.2. Let $q$ be a classical solution of the Cauchy problem (1.2), (1.3) obeying the boundedness condition (1.6). Then, for each $x \in \mathbb{R}$ and $z \in \mathbb{C} \backslash \mathbb{R}$, the Weyl $M$-matrix is differentiable in $t \in[0, T]$ and obeys

$$
\partial_{t} M=P M+M P^{\top} .
$$

Proof. For fixed $z \in \mathbb{C}_{+}$, since the time evolution of $\psi_{ \pm}$and $\partial_{x} \psi_{ \pm}$is given by (2.5), the time evolution of $m_{ \pm}$is computed as

$$
\partial_{t} m_{ \pm}= \pm \frac{\partial_{t} \partial_{x} \psi_{ \pm}}{\psi_{ \pm}}-m_{ \pm} \frac{\partial_{t} \psi_{ \pm}}{\psi_{ \pm}}= \pm P_{21}+\left(P_{22}-P_{11}\right) m_{ \pm} \mp P_{12} m_{ \pm}^{2}
$$

Then, the time derivatives of $m_{1}=\frac{-1}{m_{+}+m_{-}}, m_{2}=\frac{m_{-} m_{+}}{m_{+}+m_{-}}, m_{3}=\frac{1}{2} \frac{m_{-}-m_{+}}{m_{+}+m_{-}}$follow by mere calculations,

$$
\begin{aligned}
\partial_{t} m_{1} & =2 P_{11} m_{1}+2 P_{12} m_{3}, \\
\partial_{t} m_{2} & =-2 P_{11} m_{2}+2 P_{21} m_{3},
\end{aligned}
$$




$$
\partial_{t} m_{3}=P_{21} m_{1}+P_{12} m_{2} .
$$

So by direct computation, we have $\partial_{t} M=P M+M P^{\top}$.

Corollary 2.3. Let $q$ be a classical solution of the Cauchy problem (1.2), (1.3) obeying the boundedness condition (1.6). Then the spectrum $S=\sigma\left(H_{q(\cdot, t)}\right)$ is independent of $t \in[0, T]$ and (2.6) holds for all $z \in \mathbb{C} \backslash S$.

Proof. We use a characterization of the spectrum of a Schrödinger operator in terms of its Weyl $M$-matrix: the spectrum of $H_{q(\cdot, t)}$ is the complement of the maximal set $\Omega \subset \mathbb{C}$ such that $M(0, t ; z)$ has an analytic extension as a matrix-valued function on $\Omega$ with the "symmetry"

$$
M(0, t ; z)=M(0, t ; \bar{z})^{*} .
$$

We will show that the existence of such an analytic extension on some set is an invariant for the time evolution (2.6).

Since $M(0,0 ; z)$ has an analytic extension with the property (2.7) on $\mathbb{C} \backslash \sigma\left(H_{V}\right)$, and the matrix $P$ is bounded for $t \in[0, T]$ and analytic in $z \in \mathbb{C}$, solving (2.6) as an initial value problem starting from $t=0$ shows that $M(0, t ; z)$ is also analytic on $\mathbb{C} \backslash \sigma\left(H_{V}\right)$. Moreover, since $P(\bar{z})=\overline{P(z)}$, (2.6) implies

$$
\partial_{t} M(0, t ; \bar{z})^{*}=P(0, t ; z) M(0, t ; \bar{z})^{*}+M(0, t ; \bar{z})^{*} P(0, t ; z)^{\top}
$$

In words, $M(0, t ; \bar{z})^{*}$ obeys the same time evolution as $M(0, t ; z)$. Since $M(0,0 ; \bar{z})^{*}=M(0,0 ; z)$, it follows that $M(0, t ; \bar{z})^{*}=M(0, t ; z)$ for all $t$.

Since we have proved that $M(0, t ; z)$ has an analytic extension with the property (2.7) on $\mathbb{C} \backslash$ $\sigma\left(H_{V}\right)$, it follows that $\sigma\left(H_{q(\cdot, t)}\right) \subset \sigma\left(H_{V}\right)$ for all $t \in[0, T]$. Analogously, solving (2.6) backward in time shows that $\sigma\left(H_{V}\right) \subset \sigma\left(H_{q(\cdot, t)}\right)$ and completes the proof.

As Herglotz functions, $m_{ \pm}$have nontangential boundary values $m_{ \pm}(\lambda+i 0)$ for Lebesgue-a.e. $\lambda \in \mathbb{R}$. Moreover, for a nontrivial Herglotz function, the boundary values are nonzero almost everywhere. In particular, for Lebesgue a.e. $\lambda$, the boundary values $m_{ \pm}(\lambda+i 0)$ exist and $\left(m_{-}+\right.$ $\left.m_{+}\right)(\lambda+i 0) \neq 0$.

This justifies the definition, for a.e. $\lambda \in \sigma\left(H_{W}\right)$, of the left and right reflection coefficient for a Schrödinger operator $H_{W}$ defined as in [30] by

$$
R_{ \pm}(x ; \lambda):=\left(-\frac{m_{\mp}+\overline{m_{ \pm}}}{m_{-}+m_{+}}\right)(x ; \lambda+i 0) .
$$

If this quantity vanishes for Lebesgue-a.e. $\lambda \in \sigma\left(H_{W}\right)$, then (1.5) holds and $H_{W}$ is reflectionless. Invariance of the reflectionless property under the translation flow was already proven in [30, Corollary 3]. We note the generalization of the argument of [30, Theorem 2] to the KdV-n flow. In light of the above corollary, we may define the reflection coefficient of $H_{q(\cdot, t)}$ as

$$
R_{ \pm}(x, t ; \lambda):=\left(-\frac{m_{\mp}+\overline{m_{ \pm}}}{m_{-}+m_{+}}\right)(x, t ; \lambda+i 0)
$$

for almost very $\lambda \in \sigma\left(H_{V}\right)$. We use Lemma 2.2 to derive an ODE for the reflection coefficient.

Proposition 2.4. Suppose q obeys (1.2), (1.3), (1.6) for some $T>0$, then

$$
R_{ \pm}(x, t ; \lambda)=R_{ \pm}(x, 0 ; \lambda) \exp \left\{2 i \int_{0}^{t} \operatorname{Im}\left(m_{ \pm}(x, s ; \lambda+i 0)\right) P_{12}(x, s ; \lambda) \mathrm{d} s\right\}
$$

for all $0 \leq t \leq T$ and almost every $\lambda \in \sigma\left(H_{V}\right)$.

Proof. In addition to $z \in \mathbb{C} \backslash \sigma\left(H_{V}\right)$, the time evolution (2.6) holds wherever the Weyl $m$-functions have nontangential imits. Again, by basic properties of Herglotz functions, the nontangential limit, denoted $m_{ \pm}(x, 0, \lambda+i 0)$ exists for Lebesgue almost every $\lambda \in \mathbb{R}$. So, $m_{ \pm}(x, 0 ; \lambda+i 0)$ exists for Lebesgue almost every $\lambda \in \sigma\left(H_{V}\right)$ and the evolution in (2.2) may be extended to these $\lambda$. 
Thus, using 2.2 and the symmetry $P(\bar{z})=\overline{P(z)}$, we compute for almost every $\lambda \in \sigma\left(H_{V}\right)$,

$$
\partial_{t} R_{ \pm}(x, t ; \lambda)=2 i P_{12}(x, t ; \lambda) R_{ \pm}(x, t, \lambda) \operatorname{Im} m_{ \pm}(x, t ; \lambda+i 0)
$$

which implies (2.10).

In particular, since the reflectionless property corresponds to the case $R_{ \pm}=0$, the proof of Prop. 1.1 is immediate.

Using the results above, we will prove Cor. 1.2. Our proof relies on the following facts established in [40, 34]. By [40, if $S$ is the spectrum of a periodic operator with period $\ell$, we may associate to any point $\left\{\mu_{j}, \sigma_{j}\right\}_{j \in J} \in \mathcal{D}(S)$ a periodic operator $H_{Q}$ with the same Dirichlet data and period $\ell$. Since $H_{Q}$, being periodic, is reflectionless, the bijective correspondence between $\mathcal{R}(S)$ and $\mathcal{D}(S)$ established in [34] proves that $H_{Q}$ is the only reflectionless operator corresponding to $\left\{\mu_{j}, \sigma_{j}\right\}_{j \in J}$.

Proof of Cor. 1.2. Let $S=\sigma(V)$ and fix $t \in[0, T]$. By (2.3), $\sigma(q(\cdot, t))=S$. Associate to $q(\cdot, t)$ a point $\left\{\mu_{j}(\cdot, t), \sigma_{j}(\cdot, t)\right\} \in \mathcal{D}(S)$. By [40, there exists an operator $H_{Q(\cdot, t)}$ with Dirichlet data $\left\{\mu_{j}(\cdot, t), \sigma_{j}(\cdot, t)\right\} \in \mathcal{D}(S)$ and such that $Q(\cdot+\ell, t)=Q(\cdot, t)$. By Prop. 1.1, $q(\cdot, t) \in \mathcal{R}(S)$. Furthermore, by the bijection established in [34], and since $Q(\cdot, t) \in \mathcal{R}(S)$, we have the equality $q(\cdot, t)=Q(\cdot, t)$. Thus, $q(\cdot+\ell, t)=q(\cdot, t)$ for all $t$, so $q$ is a spatially periodic solution of $\mathrm{KdV}-n$, which implies $q(\cdot, t)=\mathcal{S}_{t}(V)$.

\section{Diagonal Green's function and trace formulas}

In order to turn our attention to Dirichlet eigenvalues, we have to review some facts about the diagonal Green's function. Consider the Schrödinger operator $H_{q(\cdot, t)}$; denote its Weyl solutions by $\psi_{ \pm}(x, t ; z)$ and $m$-functions by $m_{ \pm}(x, t ; z)$. Its diagonal Green's function can be expressed in terms of Weyl solutions as

$$
G(x, x, t ; z)=-\frac{1}{m_{-}(x, t ; z)+m_{+}(x, t ; z)} .
$$

It is well known (see [32, Theorem 4.5] and [23]) that the Weyl $m$-functions for a potential $W$ which is $2 n+1$ times differentiable in $x$ have an asymptotic expansion of the form

$$
m_{ \pm}\left(x, t ;-k^{2}\right)=-k+\sum_{j=1}^{2 n+1}( \pm 1)^{j} c_{j+1}(x, t) k^{-j}+o\left(k^{-2 n-1}\right)
$$

as $k \rightarrow \infty$ nontangentially, $\epsilon<\arg (k)<\frac{\pi}{2}-\epsilon$ for some $\epsilon>0$. The coefficients $c_{j}$ can be computed using the Ricatti equation for $m_{ \pm}$; likewise, the diagonal Green's function is found to have a similar expansion and its coefficients can be computed [22, Equation D.22] using the identity

$$
-2 G \partial_{x}^{2} G+\left(\partial_{x} G\right)^{2}+4(q-z) G^{2}=1
$$

which results in the asymptotic expansion

$$
G\left(x, x, t ;-k^{2}\right)=\frac{1}{2} \sum_{\ell=0}^{n+1} \hat{f}_{\ell} k^{-2 \ell-1}+o\left(k^{-2 n-3}\right)
$$

valid as $k \rightarrow \infty$ nontangentially, $\epsilon<\arg (k)<\frac{\pi}{2}-\epsilon$ for some $\epsilon>0$. Here $\hat{f}_{\ell}$ are precisely the quantities defined recursively in the introduction.

We will assume from now on that $S$ obeys the moment condition (1.15). We consider the scalar fields $R_{m}$ on $\mathcal{D}(S)$ for $m \leq n$. Our goal will be to prove that these are continuous scalar fields on $\mathcal{D}(S)$ and that they correspond precisely to $\hat{f}_{\ell}$ for our solution. In other words, we will prove that our solution $q(x, t)$ obeys certain trace formulas. 
We will use the following estimate from [2, Lemma 4.1]: if $S$ obeys the condition (1.15), then $Q_{k}$ is a continuous scalar field on $\mathcal{D}(S)$ for any $k \leq n+1$. It is bounded by

$$
Q_{k} \leq|\underline{E}|^{k}+3 D_{k} \sum_{j \in J}\left(1+\eta_{j, 0}^{k-1}\right) \gamma_{j}
$$

where the constant $D_{k}$ depends only on the index $k, \underline{E}$ and $\sup _{j \in J} \gamma_{j}$.

It follows that $R_{m}$ defined by (1.18) is also a continuous scalar field on $\mathcal{D}(S)$ for $1 \leq m \leq n$. This definition will be motivated by the fact that for solutions $q(x, t)$ of the Cauchy problem, the trace formulas will be of the form $\hat{f}_{m}=R_{m}$, in the sense given in Lemma 3.2 below. We begin with an elementary inequality (which will be repeatedly useful) and then proceed to derive some estimates on these scalar fields.

Lemma 3.1. For $\sigma\left(H_{V}\right)$ obeying (1.15), there are constants $M_{1}, M_{3}$ such that for $1 \leq m \leq n$,

$$
\left|R_{m}\right| \leq M_{1}
$$

and for any $k \in J$,

$$
\left|\frac{\partial R_{m}}{\partial \varphi_{k}}\right| \leq M_{3}\left(1+\left(\eta_{k, 0}+\gamma_{k}\right)^{m}\right) \gamma_{k}
$$

Proof. Note that $R_{m}$ is a polynomial in the $Q_{k}$. For the sake of clarity, we denote for $1 \leq m \leq n$,

$$
R_{m}=P_{m}\left(Q_{1}, \ldots, Q_{m}\right)
$$

In the above, the $P_{m}$ are polynomials of degree $m$ in the first $m$ scalar fields defined in (1.17). Due to the moment condition (1.15), the $Q_{k}$ for $1 \leq k \leq n+1$ are continuous, and thus, so are the $R_{m}$. Also by condition (1.15), $\mathcal{D}(S)$ with the metric (1.16) is compact. Thus, we have a uniform bound on each $R_{m}$ for $1 \leq m \leq n$. Taking the maximum of these yields $M_{1}$.

Noting that

$$
n \geq \max _{1 \leq k, j \leq n} \operatorname{deg}\left(\frac{\partial P_{k}}{\partial Q_{j}}\right)
$$

we write

$$
\frac{\partial P_{m}}{\partial Q_{j}}=\sum_{|\alpha| \leq m} A_{\alpha} \prod_{k=1}^{m} Q_{k}^{\alpha_{k}}
$$

We then take $A$ to be the maximum of all the coefficients of the $\frac{\partial P_{m}}{\partial Q_{j}}$ for $1 \leq m, j \leq n$ and using the notation in estimate (3.2), define $C=\max _{1 \leq k \leq n} D_{k}$ for convenience. We also note the elementary fact $1+x^{k} \leq 2\left(1+x^{n}\right)$ for $0 \leq k \leq n$, and $x>0$. Then for any $m$ and $j$ we have the estimate, uniform in $m$ and $j$,

$$
\begin{aligned}
\left|\frac{\partial P_{m}}{\partial Q_{j}}\right| & \leq A \sum_{|\alpha| \leq m} \prod_{k=1}^{m}\left(|\underline{E}|^{k}+3 D_{k} \sum_{i \in J}\left(1+\eta_{i, 0}^{k-1}\right) \gamma_{i}\right)^{\alpha_{k}} \\
& \leq A \sum_{|\alpha| \leq m} \prod_{k=1}^{m} 3^{\alpha_{k}}\left(1+|\underline{E}|^{n}\right)^{\alpha_{k}}\left(1+D_{k} \sum_{i \in J}\left(1+\eta_{i, 0}^{k-1}\right) \gamma_{i}\right)^{\alpha_{k}} \\
& \leq 3^{n}\left(1+|\underline{E}|^{n}\right)^{n} \sum_{|\alpha| \leq m} \prod_{k=1}^{m}\left(1+2 C \sum_{i \in J}\left(1+\eta_{i, 0}^{n-1}\right) \gamma_{i}\right)^{\alpha_{k}} \\
& \leq 3^{n}\left(1+|\underline{E}|^{n}\right)^{n}\left(1+2 C \sum_{i \in J}\left(1+\eta_{i, 0}^{n-1}\right) \gamma_{i}\right)^{n}\left(\left(\begin{array}{c}
2 n \\
n
\end{array}\right)-1\right) \\
& =: M_{2}
\end{aligned}
$$


Thus, for $1 \leq m \leq n$ we have

$$
\begin{aligned}
\left|\frac{\partial R_{m}}{\partial \varphi_{k}}\right| & =\left|\frac{\partial P_{m}}{\partial Q_{1}} \frac{\partial Q_{1}}{\partial \varphi_{k}}+\cdots+\frac{\partial P_{m}}{\partial Q_{m}} \frac{\partial Q_{m}}{\partial \varphi_{k}}\right| \\
& \leq\left|\frac{\partial P_{m}}{\partial Q_{1}}\right|\left|\frac{\partial Q_{1}}{\partial \varphi_{k}}\right|+\cdots+\left|\frac{\partial P_{m}}{\partial Q_{m}}\right|\left|\frac{\partial Q_{m}}{\partial \varphi_{k}}\right| \\
& \leq M_{2} \gamma_{k}\left(1+2\left(|\underline{E}|+\eta_{k, 0}+\gamma_{k}\right)+\cdots+m\left(|\underline{E}|+\eta_{k, 0}+\gamma_{k}\right)^{m-1}\right) \\
& \leq \frac{m(m+1)}{2}\left(1+\left(|\underline{E}|+\eta_{k, 0}+\gamma_{k}\right)^{m}\right) M_{2} \gamma_{k} .
\end{aligned}
$$

By using the power mean inequality

$$
1+\left(|\underline{E}|+\eta_{k, 0}+\gamma_{k}\right)^{m} \leq 2^{m-1}\left(1+|\underline{E}|^{m}\right)\left(1+\left(\eta_{k, 0}+\gamma_{k}\right)^{m}\right)
$$

this implies (3.3) with $M_{3}:=\frac{n(n+1)}{2} 2^{n}\left(1+|\underline{E}|^{n}\right) M_{2}$.

For a reflectionless solution $q$, let us define for each $(x, t) \in \mathbb{R} \times[0, T]$ the set of Dirichlet data $\varphi(x, t) \in \mathcal{D}(S)$. Begin by defining

$$
\mu_{j}(x, t)= \begin{cases}z \in\left(E_{j}^{-}, E_{j}^{+}\right), & G(x, x, t ; z)=0 \\ E_{j}^{+}, & G(x, x, t ; z)<0 \forall z \in\left(E_{j}^{-}, E_{j}^{+}\right) \\ E_{j}^{-}, & G(x, x, t ; z)>0 \forall z \in\left(E_{j}^{-}, E_{j}^{+}\right)\end{cases}
$$

If $\mu_{j}(x, t) \in\left(E_{j}^{-}, E_{j}^{+}\right)$, we also define $\sigma_{j}=-\left.\partial_{x} G(x, x, t ; z)\right|_{z=\mu_{j}(x, t)} \in\{ \pm 1\}$. Then $\varphi_{j}(x, t)$ are defined by (1.9), (1.10).

We will now prove that the values of differential polynomials $\hat{f}_{\ell}$ evaluated at some $(x, t)$ are precisely given by the scalar fields $R_{\ell}$ evaluated at the Dirichlet data $\varphi(x, t)$.

Lemma 3.2. Let $q$ be a classical solution of the Cauchy problem (1.2), (1.3) obeying the boundedness condition (1.6). Let $V \in \mathcal{R}(S)$ where $S$ obeys the moment condition (1.15). For all $1 \leq m \leq n$ and all $(x, t) \in \mathbb{R} \times[0, T]$,

$$
\hat{f}_{m}(x, t)=R_{m}(\varphi(x, t)) .
$$

These are higher-order trace formulas; the case $m=1$ is precisely (1.26). We also emphasize that the above lemma represents $\hat{f}_{n}$ as a polynomial in the moments $Q_{k}$.

Proof. As noted [19, Equation 2.7], the diagonal Green's function has an exponential Herglotz representation. The representation takes the form

$$
G(x, x, t ; z)=\frac{1}{2 \sqrt{z-\underline{E}}} e^{-\int_{\underline{E}}^{\infty} \frac{f(\xi)}{\xi-z} \mathrm{~d} \xi}
$$

again with the positive real axis is chosen as the branch cut for the square root, and where

$$
f(\xi)=\frac{1}{2}-\frac{1}{\pi} \arg G(x, x, t ; \xi+i 0) .
$$

The reflectionless property of the potential yields

$$
f(\xi)= \begin{cases}0, & \xi \in S \\ -\frac{1}{2}, & \xi \in\left(E_{j}^{-}, \mu_{j}\right) \\ \frac{1}{2}, & \xi \in\left(\mu_{j}, E_{j}^{+}\right)\end{cases}
$$

By the moment condition (1.15), for $k=1, \ldots, n+1$,

$$
2 k \int_{\underline{E}}^{\infty} \xi^{k-1} f(\xi) d \xi=Q_{k}-\underline{E}^{k}
$$


Assuming $\epsilon<\arg (z-\underline{E})<2 \pi-\epsilon$ for some $\epsilon>0$ and $\xi \geq \underline{E}$, we can estimate

$$
\left|\frac{1}{\xi-z}+\sum_{k=1}^{n} \frac{\xi^{k-1}}{z^{k}}\right|=\left|\frac{\frac{\xi^{n}}{z^{n}}}{\xi-z}\right| \leq\left(1+\frac{1}{\sin \epsilon}\right)\left|\frac{\xi^{n}}{z^{n}(z-\underline{E})}\right| .
$$

Multiplying by $f(\xi)$ and integrating implies that

$$
\left|\int_{\underline{E}}^{\infty} \frac{1}{\xi-z} f(\xi) \mathrm{d} \xi+\sum_{k=1}^{n} \frac{Q_{k}-\underline{E}^{k}}{2 k z^{k}}\right| \leq\left(1+\frac{1}{\sin \epsilon}\right) \frac{\left|Q_{n+1}-\underline{E}^{n+1}\right|}{|z|^{n}|z-\underline{E}|},
$$

which in turn yields the asymptotic expansion

$$
G(x, x, t ; z)=\frac{1}{2 \sqrt{z-\underline{E}}} e^{\sum_{k=1}^{n} \frac{Q_{k}-E^{k}}{2 k z^{k}}+o\left(z^{-n}\right)}
$$

as $z \rightarrow \infty, \arg (z-\underline{E}) \in(\epsilon, 2 \pi-\epsilon)$.

In order to compare coefficients to the expansion in (3.1), we write

$$
\frac{1}{2 \sqrt{z-\underline{E}}}=\frac{1}{2 \sqrt{z}} e^{-\frac{1}{2} \log \left(1-\frac{E}{z}\right)}=\frac{1}{2 \sqrt{z}} e^{\sum_{k=1}^{n} \frac{\underline{E}^{k}}{2 k z^{k}}+o\left(z^{-n}\right)}
$$

and combine with (3.7) to find

$$
\begin{aligned}
G(x, x, t ; z) & =\frac{1}{2 \sqrt{z}} e^{\sum_{k=1}^{n} \frac{Q_{k}}{2 k z^{k}}+o\left(z^{-n}\right)} \\
& =\frac{1}{2} \sum_{m=0}^{n} \frac{1}{z^{m+\frac{1}{2}}} \sum_{\substack{\alpha \in \mathbb{N}_{0}^{n} \\
\sum_{k=1}^{m} k \alpha_{k}=m}} \prod_{k=1}^{n} \frac{Q_{k}^{\alpha_{k}}}{\alpha_{k} !(2 k)^{\alpha_{k}}}+o\left(z^{-n-\frac{1}{2}}\right)
\end{aligned}
$$

Comparing coefficients to the expansion in (3.1) completes the proof.

The equation (3.6) also yields a product formula for the diagonal Green's function common in the literature:

$$
G(x, x, t ; z)=\frac{1}{2} \sqrt{\frac{1}{\underline{E}-z} \prod_{\ell \in J} \frac{\left(\mu_{\ell}(x, t)-z\right)^{2}}{\left(E_{\ell}^{-}-z\right)\left(E_{\ell}^{+}-z\right)}} .
$$

\section{Time evolution of Dirichlet eigenvalues}

Now we will derive the time evolution for $\varphi_{j}$ in terms of a Lipschitz vector field on the torus $\mathcal{D}(S)$ equipped with the metric (1.16).

Lemma 4.1. Suppose $q(x, t)$ a solution to (1.2), (1.3) satisfying (1.6). Let $\left(\varphi_{j}(x, t)\right)_{j \in J}$ be the corresponding Dirichlet data defined in (1.8), (1.9) and (1.10). If $(x, t)$ is such that $\varphi_{j}(x, t) \notin \pi \mathbb{Z}$, then

$$
\partial_{t} \varphi_{j}(x, t)=\Xi_{j}(\varphi(x, t)) .
$$

Proof. By the definitions (1.9), (1.10) and (1.8), if $\varphi_{j}(x, t) \notin \pi \mathbb{Z}$, then

$$
G\left(x, x, t ; \mu_{j}(x, t)\right)=0
$$

so by the implicit function theorem,

$$
\frac{\partial \mu_{j}}{\partial t}=-\frac{\partial G(x, x, t ; z) /\left.\partial t\right|_{z=\mu_{j}(x, t)}}{\partial G(x, x, t ; z) /\left.\partial z\right|_{z=\mu_{j}(x, t)}} .
$$


Using (1.9) and (1.10) we have differentiability of $\varphi_{j}$ and the explicit expression

$$
\frac{\partial \varphi_{j}}{\partial t}=\frac{\partial \mu_{j} / \partial t}{-\sigma_{j}(x, t) \sqrt{\left(E_{j}^{+}-\mu_{j}\right)\left(\mu_{j}-E_{j}^{-}\right)}} .
$$

Noting that $G(x, x, t ; z)=m_{1}$, the upper left entry of the Weyl $M$-matrix $M(x, t ; z)$, we read off the time evolution for the diagonal Green's function from Lemma 2.2 ,

$$
\partial_{t} m_{1}(x, t, z)=2 P_{12} m_{3}(x, t, z)+2 P_{11} m_{1}(x, t, z) .
$$

Then, plugging in $z=\mu_{j}(x, t) \in \mathbb{C} \backslash S$ in the above we see

$$
\partial_{t} m_{1}\left(x, t, \mu_{j}(x, t)\right)=-\sigma_{j}(x, t) \hat{F}_{n}\left(\mu_{j}(x, t)\right) .
$$

Differentiating the product formula (3.8) in $z$, we find

$$
\partial G(x, x, t ; z) /\left.\partial z\right|_{z=\mu_{j}(x, t)}=\frac{1}{2} \sqrt{\frac{1}{\left(\underline{E}-\mu_{j}\right)\left(E_{j}^{-}-\mu_{j}\right)\left(E_{j}^{+}-\mu_{j}\right)} \prod_{l \neq j} \frac{\left(\mu_{l}-\mu_{j}\right)^{2}}{\left(E_{l}^{-}-\mu_{j}\right)\left(E_{l}^{+}-\mu_{j}\right)}} .
$$

So, in combination, we have

$$
\frac{\partial \varphi_{j}}{\partial t}=\left(\sum_{\ell=0}^{n} R_{n-\ell}\left(\mu_{j}(x, t)\right)^{\ell}\right) \Psi_{j}
$$

We now note that by arguments identical to those found in [2, Lemma 3.4, Lemma 3.5] show that the above evolution equation for $\varphi_{j}$ holds also for $\varphi_{j}(x, t) \in \pi \mathbb{Z}$, i.e. at the gap edges. Indeed, Lemma 3.4 of [2] relies only on continuity of $G(t, x, x, z)$ in $z$ and $t$ to prove $\varphi_{j}(x, t)$ is continuous in $t$. Lemma 3.5 of [2] is an intermediate result which relies only on the continuity of the vector field $\Xi_{j}$ in time. This hypothesis is clearly satisfied in our case once we note that each $R_{n}$ is a polynomial in the $Q_{n}$, which are continuous in the $\mu_{j}$ by (1.20). In conclusion, we have the following result.

Proposition 4.2. Suppose $q(x, t)$ a solution to (1.2), (1.3) satisfying (1.6). Let $\left(\varphi_{j}(x, t)\right)_{j \in J}$ be the corresponding Dirichlet data defined in (1.8), (1.9) and (1.10). For any $(x, t) \in \mathbb{R}^{2}$ and $j \in J$,

$$
\partial_{t} \varphi_{j}(x, t)=\Xi_{j}(\varphi(x, t)) .
$$

Now that we know (4.2) holds for all $x, t$, we can view this collection of equations indexed by $j$ as an autonomous system of ODEs on $\mathcal{D}(S)$, generated by the vector field $\Xi$. We now establish sufficient conditions for this vector field to be Lipschitz on the torus: this will allow us to conclude uniqueness of solution of (4.2); as is already established, the solution to (1.2), (1.3) is uniquely recovered from the Dirichlet data.

Lemma 4.3. If the set $S=\sigma\left(H_{V}\right)$ obeys (1.20), (1.21), (1.22), (1.23), the vector field $\Xi$ with components defined by (1.19) is a Lipschitz vector field on $\mathbb{T}^{J}$.

Proof. Computing directly,

$$
\begin{aligned}
& \frac{\partial \Xi_{j}}{\partial \varphi_{k}}=\left(\sum_{\ell=0}^{n-1} \frac{\partial R_{n-\ell}}{\partial \varphi_{k}} \mu_{j}^{\ell}\right) \Psi_{j}+\left(\sum_{\ell=0}^{n} R_{n-\ell} \mu_{j}^{\ell}\right) \frac{\partial \Psi_{j}}{\partial \varphi_{k}}, \quad k \neq j \\
& \frac{\partial \Xi_{j}}{\partial \varphi_{j}}=\left(\sum_{\ell=0}^{n-1} \frac{\partial R_{n-\ell}}{\partial \varphi_{j}} \mu_{j}^{\ell}+\sum_{\ell=1}^{n-1} \ell R_{n-\ell} \frac{\partial \mu_{j}}{\partial \varphi_{j}} \mu_{j}^{\ell-1}\right) \Psi_{j}+\left(\sum_{\ell=0}^{n} R_{n-\ell} \mu_{j}^{\ell}\right) \frac{\partial \Psi_{j}}{\partial \varphi_{j}}
\end{aligned}
$$


We will now show that $\left|\Psi_{j}\right| \leq 2 C_{j}$; this follows from maximizing the smooth function $f(s)=$ $\frac{\left(E_{\ell}^{+}-\mu_{j}\right)\left(E_{\ell}^{-}-\mu_{j}\right)}{\left(E_{\ell}^{-}+s \gamma_{\ell}-\mu_{j}\right)^{2}}$ for $0 \leq s \leq 1$. For $E_{j}^{+}<E_{\ell}^{-}$, we see that $f$ decreases and,

$$
\frac{\left(E_{\ell}^{-}-\mu_{j}\right)\left(E_{\ell}^{+}-\mu_{j}\right)}{\left(\mu_{j}-\mu_{\ell}\right)^{2}} \leq \frac{E_{\ell}^{+}-\mu_{j}}{E_{\ell}^{-}-\mu_{j}}=1+\frac{\gamma_{\ell}}{E_{\ell}^{-}-\mu_{j}} \leq 1+\frac{\gamma_{\ell}}{\eta_{j, \ell}},
$$

and similarly for $E_{j}^{-}>E_{\ell}^{+}$, where $f$ increases. And, thus we have our bound on $\Psi_{j}$,

$$
\begin{aligned}
\Psi_{j} & =2 \sqrt{\left(\mu_{j}-\underline{E}\right) \prod_{\ell \neq j} \frac{\left(E_{\ell}^{-}-\mu_{j}\right)\left(E_{\ell}^{+}-\mu_{j}\right)}{\left(\mu_{j}-\mu_{\ell}\right)^{2}}} \\
& \leq 2\left(\eta_{j, 0}+\gamma_{j}\right)^{1 / 2} \prod_{\ell \neq j}\left(1+\frac{\gamma_{\ell}}{\eta_{j, \ell}}\right)^{1 / 2} \\
& =2 C_{j} .
\end{aligned}
$$

We will also need that $\left|\frac{\partial \Psi_{j}}{\partial \varphi_{k}}\right| \leq \frac{\gamma_{k}}{2 \eta_{j, k}} C_{j}$ when $k \neq j$. Note that $\left|\frac{\partial \mu_{k}}{\partial \varphi_{k}}\right| \leq \frac{1}{2} \gamma_{k}$ by a differentiation of (1.9). Then we estimate,

$$
\begin{aligned}
\Psi_{j} & =2 \sqrt{\left(\mu_{j}-\underline{E}\right) \prod_{\ell \neq j, k} \frac{\left(E_{\ell}^{-}-\mu_{j}\right)\left(E_{\ell}^{+}-\mu_{j}\right)}{\left(\mu_{j}-\mu_{\ell}\right)^{2}}} \cdot \sqrt{\frac{\left(E_{k}^{-}-\mu_{j}\right)\left(E_{k}^{+}-\mu_{j}\right)}{\left(\mu_{j}-\mu_{k}\right)^{2}}} \\
& \Longrightarrow\left|\frac{\partial \Psi_{j}}{\partial \varphi_{k}}\right|=\left|\Psi_{j}\right| \frac{1}{\left|\mu_{j}-\mu_{k}\right|}\left|\frac{\partial \mu_{k}}{\partial \varphi_{k}}\right| \leq C_{j} \frac{\gamma_{k}}{\eta_{j, k}} .
\end{aligned}
$$

Thus, for $k \neq j$,

$$
\left|\frac{\partial \Xi_{j}}{\partial \varphi_{k}}\right| \leq 2 C_{j} \sum_{\ell=0}^{n-1}\left|\frac{\partial R_{n-\ell}}{\partial \varphi_{k}}\right|\left(|\underline{E}|+\eta_{j, 0}+\gamma_{j}\right)^{\ell}+\frac{\gamma_{k}}{\eta_{j, k}} C_{j} \sum_{\ell=0}^{n}\left|R_{n-\ell}\right|\left(|\underline{E}|+\eta_{j, 0}+\gamma_{j}\right)^{\ell} .
$$

The second sum is bounded using the estimates in Lemma 3.1 as

$$
\begin{aligned}
\frac{\gamma_{k}}{\eta_{j, k}} C_{j} \sum_{\ell=0}^{n}\left|R_{n-\ell}\right|\left(|\underline{E}|+\eta_{j, 0}+\gamma_{j}\right)^{\ell} & \leq \frac{\gamma_{k}}{\eta_{j, k}} C_{j} M_{1} \sum_{\ell=0}^{n}\left(|\underline{E}|+\eta_{j, 0}+\gamma_{j}\right)^{\ell} \\
& \leq \frac{\gamma_{k}}{\eta_{j, k}}(n+1) C_{j} M_{1}\left(1+\left(|\underline{E}|+\eta_{j, 0}+\gamma_{j}\right)^{n}\right) \\
& \leq \tilde{M}_{1}\left(1+\left(\eta_{j, 0}+\gamma_{j}\right)^{n}\right) \frac{\gamma_{k}}{\eta_{j, k}} C_{j}
\end{aligned}
$$

where $\tilde{M}_{1}:=2^{n-1}(n+1) M_{1}\left(1+|\underline{E}|^{n}\right)$ and the last step uses (3.4).

For the first sum in (4.3), we again use Lemma 3.1 and (3.4), as well as the inequality $1+x^{k} \leq$ $2\left(1+x^{n}\right)$ for $0 \leq k \leq n$, and $x>0$. Indeed, we have

$$
\begin{aligned}
2 C_{j} \sum_{\ell=0}^{n-1}\left|\frac{\partial R_{n-\ell}}{\partial \varphi_{k}}\right|\left(|\underline{E}|+\eta_{j, 0}+\gamma_{j}\right)^{\ell} & \leq 4 M_{3} \gamma_{k} C_{j}\left(1+\left(\eta_{k, 0}+\gamma_{k}\right)^{n}\right) \sum_{\ell=0}^{n-1}\left(|\underline{E}|+\eta_{j, 0}+\gamma_{j}\right)^{\ell} \\
& \leq 4 M_{3}\left(1+|\underline{E}|^{n}\right) \gamma_{k} C_{j}\left(1+\left(\eta_{k, 0}+\gamma_{k}\right)^{n}\right) \sum_{\ell=0}^{n-1} 2^{\ell}\left(1+\left(\eta_{j, 0}+\gamma_{j}\right)^{\ell}\right) \\
& \leq \tilde{M}_{3} \gamma_{k} C_{j}\left(1+\left(\eta_{k, 0}+\gamma_{k}\right)^{n}\right)\left(1+\left(\eta_{j, 0}+\gamma_{j}\right)^{n}\right)
\end{aligned}
$$


where $\tilde{M}_{3}:=2^{n+2} n M_{3}\left(1+|\underline{E}|^{n}\right)$. Combining our estimates with (4.3), and applying the power mean inequality, we see for $k \neq j$,

$$
\begin{aligned}
\left|\frac{\partial \Xi_{j}}{\partial \varphi_{k}}\right| & \leq \tilde{M}_{1} C_{j} \frac{\gamma_{k}}{\eta_{j, k}}\left(1+\left(\eta_{j, 0}+\gamma_{j}\right)^{n}\right)+\tilde{M}_{3} C_{j} \gamma_{k}\left(1+\left(\eta_{k, 0}+\gamma_{k}\right)^{n}\right)\left(1+\left(\eta_{j, 0}+\gamma_{j}\right)^{n}\right) \\
& \leq \max \left\{\tilde{M}_{1}, \tilde{M}_{3}\right\} C_{j}\left(1+\left(\eta_{j, 0}+\gamma_{j}\right)^{n}\right)\left(\frac{\gamma_{k}}{\eta_{j, k}}+\gamma_{k}\left(1+\left(\eta_{k, 0}+\gamma_{k}\right)^{n}\right)\right) \\
& \leq 2^{n-1} \max \left\{\tilde{M}_{1}, \tilde{M}_{3}\right\} C_{j}\left(1+\eta_{j, 0}^{n}+\gamma_{j}^{n}\right)\left(\frac{\gamma_{k}}{\eta_{j, k}}+\gamma_{k}\left(1+2^{n-1}\left(\eta_{k, 0}^{n}+\gamma_{k}^{n}\right)\right)\right) \\
& \leq 2^{2 n-2} \max \left\{\tilde{M}_{1}, \tilde{M}_{3}\right\}\left(1+\sup _{m \in J} \gamma_{m}^{n}\right) C_{j}\left(1+\eta_{j, 0}^{n}\right)\left(\frac{\gamma_{k}}{\eta_{j, k}}+\left(1+\sup _{m \in J} \gamma_{m}^{n}\right) \gamma_{k}\left(1+\eta_{k, 0}^{n}\right)\right) \\
& \leq 2^{2 n-2} \max \left\{\tilde{M}_{1}, \tilde{M}_{3}\right\}\left(1+\sup _{m \in J} \gamma_{m}^{n}\right)^{2} C_{j}\left(1+\eta_{j, 0}^{n}\right)\left(\frac{\gamma_{k}}{\eta_{j, k}}+\gamma_{k}\left(1+\eta_{k, 0}^{n}\right)\right) \\
& \leq \tilde{M} C_{j}\left(1+\eta_{j, 0}^{n}\right)\left(\frac{\gamma_{k}}{\eta_{j, k}}+\gamma_{k}\left(1+\eta_{k, 0}^{n}\right)\right) .
\end{aligned}
$$

Where we have defined $\tilde{M}:=2^{2 n-2} \max \left\{\tilde{M}_{1}, \tilde{M}_{3}\right\}\left(1+\sup _{m \in J} \gamma_{m}^{n}\right)^{2}$ in the above for convenience. Note that $\tilde{M}$ is finite by (1.20), which implies $\sum_{m \in J} \gamma_{m}<\infty$ so that of course $\sup _{m \in J} \gamma_{m}^{n}<\infty$.

For $k=j$, in addition to the estimates above, we will need to show the following bound on $\frac{\partial \Psi_{j}}{\partial \varphi_{j}}$,

$$
\left|\frac{\partial \Psi_{j}}{\partial \varphi_{j}}\right| \leq \frac{C_{j} \gamma_{j}}{2}\left(\frac{1}{\eta_{j, 0}}+\sum_{\ell \neq j} \frac{\gamma_{\ell}}{\eta_{j, \ell}\left(\eta_{j, \ell}+\gamma_{\ell}\right)}\right)
$$

This follows by first, another maximization argument, which finds

$$
\left|\frac{2}{\mu_{\ell}-\mu_{j}}-\frac{1}{E_{\ell}^{+}-\mu_{j}}-\frac{1}{E_{\ell}^{-}-\mu_{j}}\right| \leq \frac{\gamma_{\ell}}{\eta_{j, \ell}\left(\gamma_{\ell}+\eta_{j, \ell}\right)} .
$$

The above justifies the termwise differentiation of the sum over the index $J$ and shows

$$
\begin{aligned}
\left|\frac{\partial \Psi_{j}}{\partial \varphi_{j}}\right| & =\left|\frac{\Psi_{j}}{2} \frac{\partial \mu_{j}}{\partial \varphi_{j}}\left(\frac{1}{\mu_{j}-\underline{E}}+\sum_{\ell \neq j}\left(\frac{2}{\mu_{\ell}-\mu_{j}}-\frac{1}{E_{\ell}^{+}-\mu_{j}}-\frac{1}{E_{\ell}^{-}-\mu_{j}}\right)\right)\right| \\
& \leq \frac{C_{j} \gamma_{j}}{2}\left(\frac{1}{\eta_{j, 0}}+\sum_{\ell \neq j} \frac{\gamma_{\ell}}{\eta_{j, \ell}\left(\gamma_{\ell}+\eta_{j, \ell}\right)}\right) .
\end{aligned}
$$

For the first term in $\frac{\partial \Xi_{j}}{\partial \varphi_{j}}$, we use (3.4) to find

$$
\begin{aligned}
\sum_{\ell=0}^{n-1}\left|\frac{\partial R_{n-\ell}}{\partial \varphi_{j}}\right|\left|\mu_{j}\right|^{\ell} & \leq M_{3} \gamma_{j} \sum_{\ell=0}^{n-1}\left(1+\left(\eta_{j, 0}+\gamma_{j}\right)^{n-\ell}\right)\left(|\underline{E}|+\eta_{j, 0}+\gamma_{j}\right)^{\ell} \\
& \leq 2^{n-1} M_{3}\left(1+|\underline{E}|^{n}\right) \gamma_{j} \sum_{\ell=0}^{n-1}\left(1+\left(\eta_{j, 0}+\gamma_{j}\right)^{n-\ell}\right)\left(1+\left(\eta_{j, 0}+\gamma_{j}\right)^{\ell}\right) \\
& \leq 2^{n+1} n M_{3}\left(1+|\underline{E}|^{n}\right) \gamma_{j}\left(1+\left(\eta_{j, 0}+\gamma_{j}\right)^{n}\right) \\
& =\frac{1}{2} \tilde{M}_{3} \gamma_{j}\left(1+\left(\eta_{j, 0}+\gamma_{j}\right)^{n}\right)
\end{aligned}
$$


since $\left(1+\left(\eta_{j, 0}+\gamma_{j}\right)^{n-\ell}\right)\left(1+\left(\eta_{j, 0}+\gamma_{j}\right)^{\ell}\right) \leq 4\left(1+\left(\eta_{j, 0}+\gamma_{j}\right)^{n}\right)$. Similar methods bound the remaining two terms:

$$
\begin{aligned}
\sum_{\ell=1}^{n-1} \ell\left|R_{n-\ell}\right|\left|\frac{\partial \mu_{j}}{\partial \varphi_{j}}\right|\left|\mu_{j}\right|^{\ell-1} & \leq \frac{(n-1) M_{1}}{2} \gamma_{j} \sum_{\ell=1}^{n-1}\left(|\underline{E}|+\eta_{j, 0}+\gamma_{j}\right)^{\ell-1} \\
& \leq \frac{(n-1) M_{1}}{2}\left(1+|\underline{E}|^{n}\right) \gamma_{j} \sum_{\ell=1}^{n-1} 2^{\ell-1}\left(1+\left(\eta_{j, 0}+\gamma_{j}\right)^{\ell-1}\right) \\
& \leq 2^{n-1} \frac{(n-1)^{2} M_{1}}{2}\left(1+|\underline{E}|^{n}\right) \gamma_{j}\left(1+\left(\eta_{j, 0}+\gamma_{j}\right)^{n}\right) \\
& \leq \frac{(n-1)}{2} \tilde{M}_{1} \gamma_{j}\left(1+\left(\eta_{j, 0}+\gamma_{j}\right)^{n}\right)
\end{aligned}
$$

and

$$
\begin{aligned}
\sum_{\ell=0}^{n}\left|R_{n-\ell}\right|\left|\mu_{j}\right|^{\ell} & \leq M_{1} \sum_{\ell=0}^{n}\left(|\underline{E}|+\eta_{j, 0}+\gamma_{j}\right)^{\ell} \\
& \leq M_{1}\left(1+|\underline{E}|^{n}\right) \sum_{\ell=0}^{n} 2^{\ell}\left(1+\left(\eta_{j, 0}+\gamma_{j}\right)^{\ell}\right) \\
& \leq 2^{n+1} M_{1}(n+1)\left(1+|\underline{E}|^{n}\right)\left(1+\left(\eta_{j, 0}+\gamma_{j}\right)^{n}\right) \\
& =4 \tilde{M}_{1}\left(1+\left(\eta_{j, 0}+\gamma_{j}\right)^{n}\right) .
\end{aligned}
$$

Thus, we have

$$
\begin{aligned}
\left|\frac{\partial \Xi_{j}}{\partial \varphi_{j}}\right| \leq & 2 C_{j}\left(\sum_{\ell=0}^{n-1}\left|\frac{\partial R_{n-\ell}}{\partial \varphi_{j}}\right|\left|\mu_{j}\right|^{\ell}+\sum_{\ell=1}^{n-1} \ell\left|R_{n-\ell}\right|\left|\frac{\partial \mu_{j}}{\partial \varphi_{j}}\right|\left|\mu_{j}\right|^{\ell-1}\right)+\left(\sum_{\ell=0}^{n}\left|R_{n-\ell}\right|\left|\mu_{j}\right|^{\ell}\right)\left|\frac{\partial \Psi_{j}}{\partial \varphi_{j}}\right| \\
\leq & \tilde{M}_{3} C_{j} \gamma_{j}\left(1+\left(\eta_{j, 0}+\gamma_{j}\right)^{n}\right) \\
& +(n-1) \tilde{M}_{1} C_{j} \gamma_{j}\left(1+\left(\eta_{j, 0}+\gamma_{j}\right)^{n}\right) \\
& +2 \tilde{M}_{1} C_{j} \gamma_{j}\left(\frac{1}{\eta_{j, 0}}+\sum_{k \neq j} \frac{\gamma_{k}}{\eta_{j, k}\left(\eta_{j, k}+\gamma_{k}\right)}\right)\left(1+\left(\eta_{j, 0}+\gamma_{j}\right)^{n}\right) \\
\leq & \tilde{M}_{3} 2^{n-1}\left(\sup _{m \in J} \gamma_{m}^{n}+1\right) C_{j} \gamma_{j}\left(1+\eta_{j, 0}^{n}\right) \\
& +(n-1) \tilde{M}_{1} 2^{n-1}\left(\sup _{m \in J} \gamma_{m}^{n}+1\right) C_{j} \gamma_{j}\left(1+\eta_{j, 0}^{n}\right) \\
& +2 \tilde{M}_{1} 2^{n-1}\left(\sup _{m \in J} \gamma_{m}^{n}+1\right) C_{j} \gamma_{j}\left(\frac{1}{\eta_{j, 0}}+\sum_{k \neq j} \frac{\gamma_{k}}{\eta_{j, k}\left(\eta_{j, k}+\gamma_{k}\right)}\right)\left(1+\eta_{j, 0}^{n}\right) \\
\leq & 2 n \tilde{M} C_{j} \gamma_{j}\left(1+\eta_{j, 0}^{n}\right)\left(1+\frac{1}{\eta_{j, 0}}+\sum_{k \neq j} \frac{\gamma_{k}}{\eta_{j, k}\left(\eta_{j, k}+\gamma_{k}\right)}\right)
\end{aligned}
$$

Finally, we collect the above estimates and show $\Xi$ is Lipschitz.

$$
\begin{aligned}
\|\Xi(\varphi)-\Xi(\tilde{\varphi})\| & =\sup _{j \in J} \gamma_{j}^{1 / 2}\left(1+\eta_{j, 0}^{n}\right)^{1 / 2}\left\|\Xi_{j}(\varphi)-\Xi_{j}(\tilde{\varphi})\right\| \\
& \leq \sup _{j \in J} \gamma_{j}^{1 / 2}\left(1+\eta_{j, 0}^{n}\right)^{1 / 2} \sum_{k \in J}\left\|\frac{\partial \Xi_{j}}{\partial \varphi_{k}}\right\|_{\infty}\left\|\varphi_{k}-\tilde{\varphi}_{k}\right\|
\end{aligned}
$$




$$
\begin{aligned}
& \leq\|\varphi-\tilde{\varphi}\| \sup _{j \in J} \sum_{k \in J} \gamma_{j}^{1 / 2}\left(1+\eta_{j, 0}^{n}\right)^{1 / 2} \gamma_{k}^{-1 / 2}\left(1+\eta_{k, 0}^{n}\right)^{-1 / 2}\left\|\frac{\partial \Xi_{j}}{\partial \varphi_{k}}\right\|_{\infty} \\
& \leq\|\varphi-\tilde{\varphi}\|\left(\sup _{j \in J} \gamma_{j}^{1 / 2}\left(1+\eta_{j, 0}^{n}\right)^{1 / 2} \sum_{k \neq j} \gamma_{k}^{-1 / 2}\left(1+\eta_{k, 0}^{n}\right)^{-1 / 2}\left\|\frac{\partial \Xi_{j}}{\partial \varphi_{k}}\right\|_{\infty}+\sup _{j \in J}\left\|\frac{\partial \Xi_{j}}{\partial \varphi_{j}}\right\|_{\infty}\right) .
\end{aligned}
$$

We examine the sum over $k \neq j$ first. Using our estimates on $\left\|\frac{\partial \Xi_{j}}{\partial \varphi_{k}}\right\|_{\infty}$ for $k \neq j$ computed above, we see:

$$
\begin{aligned}
& \sup _{j \in J} \gamma_{j}^{1 / 2}\left(1+\eta_{j, 0}^{n}\right)^{1 / 2} \sum_{k \neq j} \gamma_{k}^{-1 / 2}\left(1+\eta_{k, 0}^{n}\right)^{-1 / 2}\left\|\frac{\partial \Xi_{j}}{\partial \varphi_{k}}\right\|_{\infty} \\
& \leq \tilde{M} \sup _{j \in J} C_{j}\left(1+\eta_{j, 0}^{n}\right)^{3 / 2} \sum_{k \neq j} \frac{\gamma_{k}^{1 / 2} \gamma_{j}^{1 / 2}}{\eta_{j, k}\left(1+\eta_{k, 0}^{n}\right)^{1 / 2}} \\
& \quad+\tilde{M} \sup _{j \in J} C_{j} \gamma_{j}^{1 / 2}\left(1+\eta_{j, 0}^{n}\right)^{3 / 2} \sum_{k \neq j} \gamma_{k}^{1 / 2}\left(1+\eta_{k, 0}^{n}\right)^{1 / 2} \\
& \leq \tilde{M} \sup _{j \in J} C_{j}\left(1+\eta_{j, 0}^{n}\right)^{3 / 2} \sum_{k \neq j} \frac{\gamma_{k}^{1 / 2} \gamma_{j}^{1 / 2}}{\eta_{j, k}\left(1+\eta_{k, 0}^{n}\right)^{1 / 2}} \\
& \quad+\tilde{M}\left(\sum_{k \in J} \gamma_{k}^{1 / 2}\left(1+\eta_{k, 0}^{n}\right)^{1 / 2}\right) \sup _{j \in J} C_{j} \gamma_{j}^{1 / 2}\left(1+\eta_{j, 0}^{n}\right)^{3 / 2} \\
& =:
\end{aligned}
$$

where $L_{1}<\infty$ by assumption (1.21) on the first term, and (1.20) and (1.23) on the second.

For the $k=j$ term, we take a supremum over our estimate on $\frac{\partial \Xi_{j}}{\partial \varphi_{j}}$, bounding as follows,

$$
\begin{aligned}
\sup _{j \in J}\left|\frac{\partial \Xi_{j}}{\partial \varphi_{j}}\right| & \leq 2 n \tilde{M} \sup _{j \in J} C_{j} \gamma_{j}\left(1+\eta_{j, 0}^{n}\right)\left(1+\frac{1}{\eta_{j, 0}}+\sum_{k \neq j} \frac{\gamma_{k}}{\eta_{j, k}\left(\eta_{j, k}+\gamma_{k}\right)}\right) \\
& \leq 2 n \tilde{M}\left(\sup _{j \in J} C_{j} \gamma_{j}\left(1+\eta_{j, 0}^{n}\right)+\sup _{j \in J} \frac{\gamma_{j}\left(1+\eta_{j, 0}^{n}\right) C_{j}}{\eta_{j, 0}}+\sup _{j \in J} C_{j} \gamma_{j}\left(1+\eta_{j, 0}^{n}\right) \sum_{k \neq j} \frac{\gamma_{k}}{\eta_{j, k}\left(\eta_{j, k}+\gamma_{k}\right)}\right) \\
& \leq 2 n \tilde{M}\left(\sup _{j \in J} C_{j} \gamma_{j}\left(1+\eta_{j, 0}^{n}\right)+\sup _{j \in J} \frac{\gamma_{j}\left(1+\eta_{j, 0}^{n}\right) C_{j}}{\eta_{j, 0}}+\sup _{j \in J} C_{j}\left(1+\eta_{j, 0}^{n}\right) \sum_{k \neq j}\left(\frac{\gamma_{k}^{1 / 2} \gamma_{j}^{1 / 2}}{\eta_{j, k}}\right)^{2}\right) \\
& =: L_{2} .
\end{aligned}
$$

In the above, the first term in $L_{2}$ is finite by (1.23), once we note that (1.20) implies $\gamma_{j}<1$ for all but finitely many indices $j \in J$, so that for these indices, $\gamma_{j}<\gamma_{j}^{1 / 2}$. For the second term, we make use of (1.22), and for the final term, (1.21) suffices.

In summary,

$$
\|\Xi(\varphi)-\Xi(\tilde{\varphi})\| \leq\left(L_{1}+L_{2}\right)\|\varphi-\tilde{\varphi}\|
$$

and the vector field is Lipschitz.

The proof of the remainder of Theorem 1.4 is now nothing more than collecting our results.

Proof of Theorem 1.4. Let $V: \mathbb{R} \rightarrow \mathbb{R}$ be such that $H_{V}$ is reflectionless and $\sigma\left(H_{V}\right)=S$, where $S$ obeys (1.20), (1.21), (1.22), and (1.23). Associate to $V$ the angular variables $\left(\tilde{\varphi}_{j}(x)\right)_{j \in J} \in \mathcal{R}(S)$. 
Let $q(x, t)$ be a classical solution to (1.2), (1.3) satisfying boundedness condition (1.6). By Cor. 2.3 and Prop. 1.1, we may associate angular variables $\left(\varphi_{j}(x, t)\right)_{j \in J} \in \mathcal{R}(S)$ at any time $t \in[0, T]$. Using the trace formula (1.13), this allows us to write

$$
q(x, t)=\underline{E}+\sum_{j \in J}\left(E_{j}^{+}+E_{j}^{-}-2 \mu_{j}(x, t)\right)
$$

By Prop. 4.2, $\varphi_{j}(x, t)$ satisfies

$$
\partial_{t} \varphi_{j}(x, t)=\Xi_{j}(\varphi)
$$

where $\Xi$ is Lipschitz by Lemma 4.3. Thus, setting the initial condition $\left(\varphi_{j}(x, 0)\right)_{j \in J}=\left(\tilde{\varphi}_{j}(x)\right)_{j \in J}$ ensures the $\mu_{j}(x, t)$ in the above trace formula representation, and thus the solution $q(x, t)$, is unique.

\section{Application to Small Quasi-Periodic initial Data With Diophantine Frequency}

In this section, we will show our uniqueness result applies to small quasi-periodic intial data in order to prove Theorem 1.6.

In the setting of Theorem [1.6 it is natural to label the gaps by the values of the rotation number $|m \cdot \omega|$, or more concisely, by $m \in \mathbb{Z}^{\nu}$, with the labels $m$ and $-m$ corresponding to the same gap, while $m=0$ corresponds to the bottom of the spectrum. For $\epsilon<\epsilon_{0}\left(a_{0}, b_{0}, \kappa_{0}\right)$, in [12, 2], the following bounds were established:

(1) For every $m \in \mathbb{Z}^{\nu} \backslash\{0\}$,

$$
\gamma_{m}<2 \epsilon \exp \left(-\frac{\kappa_{0}}{2}|m|\right)
$$

(2) For every $m \in \mathbb{Z}^{\nu} \backslash\{0\}$,

$$
\eta_{m, 0} \leq c|m|^{2}
$$

for a constant $c$ depending only on $\epsilon$ and $\omega$.

(3) For every $m \in \mathbb{Z}^{\nu} \backslash\{0\}, n \in \mathbb{Z}^{\nu}, m \neq n$ and $|m| \geq|n|$,

$$
\eta_{m, n} \geq a|m|^{-b}
$$

for constants $a, b>0$ depending only on $\omega, \epsilon, \kappa_{0}, \nu$.

As a consequence [2], the constants $C_{m}$ defined in (1.14) obey

$$
C_{m} \leq F \exp (F \log |m| \log \log |m|)
$$

for $F=F\left(a, b, \kappa_{0}, \nu, \omega\right)$.

Our goal is to use the above estimates to show that the spectrum of $H_{V}$, for $V$ of the form (1.27), obeys the Craig-type conditions (1.20), (1.21), (1.22), (1.23), and therefore that Theorem 1.4 applies. This will prove Theorem 1.6.

Lemma 5.1. For $V$ of the form (1.27), and satisfying the Diophantine condition (1.28), conditions (1.20), (1.21), (1.22), (1.23) are satisfied for any positive integer $n$.

Proof. For (1.20), we combine (5.1) and (5.2) to find

$$
\sum_{m \in \mathbb{Z}^{\nu} \backslash\{0\}} \gamma_{m}^{1 / 2}\left(1+\eta_{m, 0}^{n}\right)^{1 / 2}<\sqrt{2 \epsilon} \sum_{m \in \mathbb{Z}^{\nu} \backslash\{0\}} \exp \left(-\frac{\kappa_{0}|m|}{4}\right)\left(1+c^{n}|m|^{2 n}\right)^{1 / 2}<\infty .
$$

For (1.21), we use (5.3) to bound the sum;

$$
\sum_{k \neq m} \frac{\gamma_{k}^{1 / 2}}{\eta_{m, k}}=\sum_{|k|>|m|} \frac{\gamma_{k}^{1 / 2}}{\eta_{m, k}}+\sum_{|k|<|m|} \frac{\gamma_{k}^{1 / 2}}{\eta_{m, k}}
$$




$$
\begin{aligned}
& \leq \frac{2 \epsilon}{a}\left(\sum_{|k|>|m|}|k|^{b} \exp \left(-\frac{\kappa_{0}|k|}{4}\right)+|m|^{b} \sum_{|k|<|m|} \exp \left(-\frac{\kappa_{0}|k|}{4}\right)\right) \\
& \leq \frac{2 \epsilon}{a}\left(\sum_{k \neq m}|k|^{b} \exp \left(-\frac{\kappa_{0}|k|}{4}\right)\right)\left(1+|m|^{b}\right) .
\end{aligned}
$$

And from (5.4), (5.2), we have for $m \in \mathbb{Z}^{\nu} \backslash\{0\}$,

$$
C_{m} \gamma_{m}^{1 / 2}\left(1+\eta_{m, 0}^{n}\right)^{3 / 2} \leq F \exp \left(F \log |m| \log \log |m|-\frac{\kappa_{0}|m|}{4}\right)\left(1+c^{n}|m|^{2 n}\right)^{3 / 2} .
$$

And by taking the product of these estimates we may conclude

$$
\sup _{m \neq 0} C_{m} \gamma_{m}^{1 / 2}\left(1+\eta_{m, 0}^{n}\right)^{3 / 2} \sum_{k \neq m} \frac{\gamma_{k}^{1 / 2}}{\eta_{m, k}}<\infty .
$$

For (1.22), it suffices to note for $m \in \mathbb{Z}^{\nu} \backslash\{0\}$,

$$
\frac{\gamma_{m}\left(1+\eta_{m, 0}^{n}\right) C_{m}}{\eta_{m, 0}} \leq \frac{2 \epsilon}{a} F \exp \left(F \log |m| \log \log |m|-\frac{\kappa_{0}}{2}|m|\right)\left(1+c^{n}|m|^{2 n}\right)|m|^{b}
$$

and so $\sup _{m \neq 0} \frac{\gamma_{m}\left(1+\eta_{m, 0}^{n}\right) C_{m}}{\eta_{m, 0}}<\infty$. (1.23) follows immediately from the estimate

$$
\gamma_{m}^{1 / 2}\left(1+\eta_{m, 0}^{n}\right)^{3 / 2} C_{m} \leq \sqrt{2 \epsilon} F \exp \left(F \log |m| \log \log |m|-\frac{\kappa_{0}}{4}|m|\right)\left(1+c^{n}|m|^{2 n}\right)^{3 / 2}
$$

for $m \in \mathbb{Z}^{\nu} \backslash\{0\}$.

Proof of Theorem 1.6. By [8], $\sigma\left(H_{V}\right)=\sigma_{\mathrm{ac}}\left(H_{V}\right)$. Thus, by Kotani theory, $H_{V}$ is reflectionless 25]. Furthermore, by Lemma 5.1, conditions (1.20), (1.21), (1.22), and (1.23) are satisfied. Thus we have, by Theorem 1.4, uniqueness of any solution $q(x, t)$ to (1.2), (1.3), provided $q, \partial_{x}^{2 n} q \in$ $L^{\infty}(\mathbb{R} \times[0, T])$.

\section{REFERENCES}

[1] A. Avila, On the spectrum and Lyapunov exponent of limit-periodic Schrödinger operators, Comm. Math. Phys. 288 (2009), 907-918.

[2] I. Binder, D. Damanik, M. Goldstein, M. Lukic, Almost Periodicity in Time of Solutions of the KdV Equation, Duke Math. J. 167 (2018), 2633-2678.

[3] I. Binder, D. Damanik, M. Lukic, T. VandenBoom, Almost Periodicity in Time of Solutions of the Toda Lattice, C. R. Math. Rep. Acad. Sci. Canada 40 (2018), 1-28.

[4] J. Boussinesq, Essai sur la theorie des eaux courantes, Memoires presentes par divers savants, l'Acad. des Sci. Inst. Nat. France, XXIII (1877), pp. 1-680.

[5] A. Boutet de Monvel, I. Egorova, On solutions of nonlinear Schrödinger equations with Cantor-type spectrum, J. Anal. Math. 72 (1997), 1-20.

[6] W. Craig, The Trace Formula for Schrödinger Operators on the Line, Comm. Math. Phys., 126(2):379-407, 1989.

[7] D. Damanik, J. Fillman, M. Lukic, Limit-Periodic Continuum Schrödinger Operators with Zero Measure Cantor Spectrum, J. Spectr. Theory 7 (2017), 1101-1118.

[8] D. Damanik, M. Goldstein, On the inverse spectral problem for the quasi-periodic Schrödinger equation, Publ. Math. Inst. Hautes Études Sci. 119 (2014), 217-401.

[9] D. Damanik, M. Goldstein, On the existence and uniqueness of global solutions of the KdV equation with quasiperiodic initial data, J. Amer. Math. Soc. 29 (2016), 825-856.

[10] D. Damanik, M. Goldstein, M. Lukic, A multi-scale analysis scheme on Abelian groups with an application to operators dual to Hill's equation, Trans. Amer. Math. Soc. 369 (2017), 1689-1755.

[11] D. Damanik, M. Goldstein, M. Lukic, The isospectral torus of quasi-periodic Schrödinger operators via periodic approximations, Invent. Math. 207 (2017), 895-980.

[12] D. Damanik, M. Goldstein, M. Lukic, The spectrum of a Schrödinger operator with small quasi-periodic potential is homogeneous, J. Spectr. Theory 6 (2016), 415-427. 
[13] D. Damanik, M. Goldstein, W. Schlag, M. Voda, Homogeneity of the spectrum for quasi-periodic Schrödinger operators, J. Eur. Math. Soc. 20 (2018), 3073-3111.

[14] P. Deift, Some open problems in random matrix theory and the theory of integrable systems. Integrable Systems and Random Matrices, 419-430, Contemp. Math. 458, Amer. Math. Soc., Providence, RI, 2008.

[15] P. Deift, Some Open Problems in Random Matrix Theory and the Theory of Integrable Systems. II, SIGMA 13 (2017), 016, 23 pages.

[16] P. Deift, Fifty Years of KdV: An Integrable System, arXiv:1902.10267.

[17] I. E. Egorova, Almost periodicity of some solutions of the KdV equation with Cantor spectrum, Dopov./Dokl. Akad. Nauk Ukraini, (1993), 26-29.

[18] I. E. Egorova, The Cauchy problem for the KdV equation with almost periodic initial data whose spectrum is nowhere dense, Adv. Soviet Math., 19 (1994), 181-208.

[19] B. Eichinger, T. Vandenboom, P. Yuditskii, KdV hierarchy via Abelian coverings and operator identities, Trans. Amer. Math. Soc. Ser. B, 6, (2019), 1-44.

[20] H. Eliasson, Floquet solutions for the 1-dimensional quasiperiodic Schrödinger equation. Commun. Math. Phys. 146 (1992), 447-482.

[21] C. S. Gardner, J. M. Greene, M. D. Kruskal, R. M. Miura, A method of solving the Korteweg-de Vries equation, Phys. Rev. Lett. 19 (1967), 1095-1097.

[22] F. Gesztesy, H. Holden, Soliton equations and their algebro-geometric solutions. Vol. I. $(1+1)$-dimensional continuous models. Cambridge University Press, Cambridge, 2003.

[23] F. Gesztesy, H. Holden, B. Simon, Z. Zhao, Higher order trace relations for Schrödinger operators, Rev. Math. Phys. 7 (1995), 893-922.

[24] D. J. Korteweg, G. de Vries, On the change of form of long waves advancing in a rectangular canal, and on a new type of long stationary waves, Philos. Mag. 539 (1895), 422-443.

[25] S. Kotani, Ljapunov indices determine absolutely continuous spectra of stationary random one-dimensional Schrödinger operators, Stochastic Analysis (Katata/Kyoto, 1982), 225-247, North-Holland Math. Library 32, North-Holland, Amsterdam, 1984

[26] P. Lax, Integrals of non-linear equations of evolution and solitary waves, Comm. Pure Appl. Math. 21 (1968), 467-490.

[27] M. Lukic, Derivatives of $L^{p}$ eigenfunctions of Schrödinger operators, Math. Model. Nat. Phenom. 8 (2013), $170-174$.

[28] T. Oh, On nonlinear Schrödinger equations with almost periodic initial data, SIAM J. Math. Anal. 47 (2015), $1253-1270$.

[29] C. Remling, Generalized reflection coefficients, Comm. Math. Phys. 337 (2015), 1011-1026.

[30] A. Rybkin, On the evolution of a reflection coefficient under the Korteweg-de Vries flow, J. Math. Phys. 49 (2008), 072701, 15 pp.

[31] B. Simon, Bounded eigenfunctions and absolutely continuous spectra for one-dimensional Schrödinger operators, Proc. Amer. Math. Soc. 124 (1996), 3361-3369.

[32] B. Simon, A new approach to inverse spectral theory, I. Fundamental formalism, Ann. of Math, 126(2):379-407, 1989.

[33] M. Sodin, P. Yuditskii, Almost periodic Jacobi matrices with homogeneous spectrum, infinite-dimensional Jacobi inversion, and Hardy spaces of character-automorphic functions, J. Geom. Anal. 7 (1997), 387-435.

[34] M. Sodin, P. Yuditskii, Almost periodic Sturm-Liouville operators with Cantor homogeneous spectrum, Comment. Math. Helv. 70 (1995), 639-658.

[35] M. Spivak, A Comprehensive Introduction to Differential Geometry. Vol. I, Publish or Perish, Inc. Houston, 2005.

[36] G. Stolz, Bounded solutions and absolute continuity of Sturm-Liouville operators, J. Math. Anal. Appl. 169 (1992), 210-228.

[37] G. Stolz, Localization for random Schrödinger operators with Poisson potential, Ann. Inst. H. Poincaré Phys. Théor. 63 (1995), no. 3, 297-314.

[38] K. Tsugawa, Local well-posedness of KdV equations with quasi-periodic initial data, SIAM Journal of Mathematical Analysis, 44 (2012), 3412-3428.

[39] V. Vinnikov, P. Yuditskii, Functional models for almost periodic Jacobi matrices and the Toda hierarchy, Mat. Fiz. Anal. Geom. 9 (2002), 206-219.

[40] V. A. Marčenko, I. O. Ostrowskii, A characterization of the spectrum of Hill's operator, Math. USSR Sbornik 97 (1975) 493-554

Department of Mathematics, Rice University, Houston, TX 77005, USA

E-mail address: milivoje.lukic@rice.edu,gfy1@rice.edu 